\title{
Variations saisonnières de la croissance diamétrique et des phénologies foliaire et reproductive de trois espèces ligneuses commerciales d'Afrique centrale
}

Fousseni FÉTÉKÉ ${ }^{1}$ Adeline FAYOLLE ${ }^{2}$ Kasso DaINOU 3 Nils BOURLAND ${ }^{4}$ Agathe DIÉ4 Philippe LeJEUNE ${ }^{1}$ Jean-Louis DouCET ${ }^{2}$ Hans BeECKMAN ${ }^{4}$

1 Université de Liège Gembloux Agro-Bio Tech Unité BIOSE

Gestion des ressources forestières Passage des Déportés, 2

5030 Gembloux Belgique

2 Université de Liège Gembloux

Agro-Bio Tech

Central African Forests

TERRA Research Centre

Passage des Déportés, 2

5030 Gembloux

Belgique

3 Nature Plus ASBL

WINSTAR Park

Rue Provinciale, 62

1301 Wavre

Belgique

4 Musée royal de l'Afrique centrale Service de biologie du bois 3080 Tervuren

Belgique

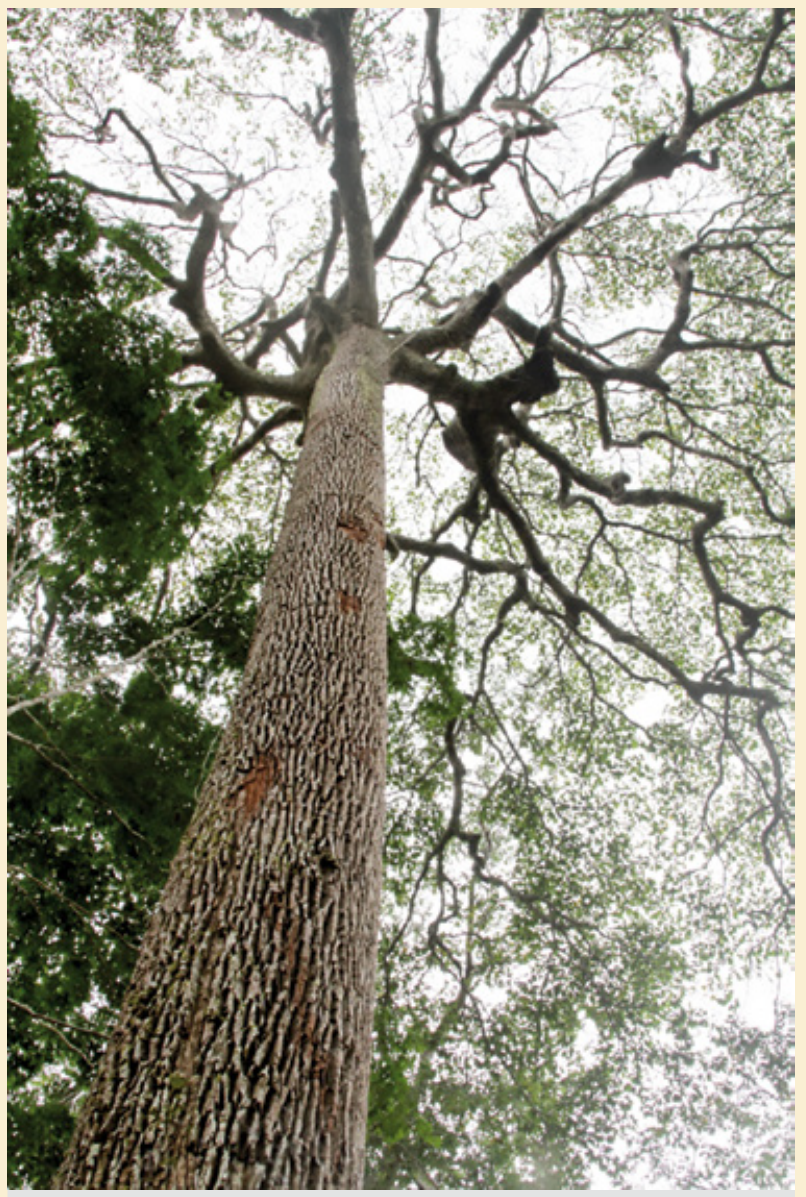

Baillonella toxisperma.

Photo J.-L. Doucet. 


\section{RÉSUMÉ}

\section{VARIATIONS SAISONNIÈRES DE LA CROISSANCE DIAMÉTRIQUE ET DES PHÉNOLOGIES FOLIAIRE ET REPRODUCTIVE DE TROIS ESPÈCES LIGNEUSES COMMERCIALES D'AFRIQUE CENTRALE}

La rythmicité intra-annuelle de la croissance diamétrique des arbres n'est connue que pour très peu d'espèces tropicales africaines. Dans cet article, une combinaison de méthodes expérimentant le marquage cambial séquentiel, l'analyse anatomique et la mesure des cernes a été utilisée pour déterminer la périodicité et les variations de la croissance diamétrique de trois espèces d'Afrique centrale produisant un bois d'œuvre de grande qualité : Baillonella toxisperma (moabi), Entandrophragma cylindricum (sapelli) et Erythrophleum suaveolens (tali). En outre, des interactions avec la phénologie foliaire et reproductive et le climat ont été recherchées. Pour les trois espèces, les cernes de croissance sont anatomiquement distincts, annuels et se forment au rythme des saisons, avec un accroissement qui est maximal pendant la petite saison pluvieuse (de mars à mai). Les variations saisonnières de la croissance se sont avérées significatives pour le moabi et le sapelli et non significatives pour le tali. Les paramètres climatiques jouant un rôle déterminant dans ces variations sont la température et l'ensoleillement. L'analyse dendrochronologique dresse d'intéressantes perspectives en vue d'acquérir des données de croissance sur le long terme, nécessaires à la définition des paramètres de gestion des populations naturelles de ces espèces.

Mots-clés : Baillonella toxisperma, Entandrophragma cylindricum, Erythrophleum suaveolens, croissance, activité cambiale, phénologie, saisonnalité des cernes, forêt semi-caducifoliée, Afrique centrale.

\section{ABSTRACT}

\section{SEASONAL VARIATIONS IN DIAMETER GROWTH AND LEAF AND REPRODUCTIVE PHENOLOGY IN THREE CENTRAL AFRICAN COMMERCIAL TIMBER SPECIES}

The intra-annual rhythms of diameter growth is known for only a very few tropical African timber species. For this study, we used several methods in combination - sequential cambium marking, anatomical analysis and growth ring measurements - to determine the periodicity and variations of diameter growth in three Central African tree species producing very high quality timber: Baillonella toxisperma (Moabi), Entandrophragma cylindricum (Sapelli) and Erythrophleum suaveolens (Tali). We also sought to identify interactions between climate and foliar and reproductive phenology. All three species have anatomically distinct annual growth rings that form as the season advances, reaching their maximum rate of growth during the short rainy season (March to May). The seasonal variations in growth rates proved to be significant in Moabi and Sapelli and not significant in Tali. The climate parameters with a determining role in these variations were temperature and hours of sunshine. The dendrochronological analysis opened up interesting prospects for the long-term acquisition of the growth data needed to define parameters for the management of natural stands of these species.

Keywords: Baillonella toxisperma, Entandrophragma cylindricum, Erythrophleum suaveolens, growth, cambium activity, phenology, seasonal growth ring development, semi-deciduous forest, Central Africa.

\section{RESUMEN}

\section{VARIACIONES ESTACIONALES DEL CRECIMIENTO DIAMETRAL Y DE LA FENOLOGÍA FOLIAR Y REPRODUCTIVA DE TRES ESPECIES LEÑOSAS COMERCIALES DE ÁFRICA CENTRAL}

La ritmicidad intraanual del crecimiento diametral de los árboles sólo se conoce en muy pocas especies tropicales africanas. En este artículo se describe la utilización de una combinación de métodos que experimentan el marcado secuencial del cambium, el análisis anatómico y la medición de los anillos para determinar la periodicidad y variaciones del crecimiento diametral de tres especies de África Central que producen una madera de gran calidad: Baillonella toxisperma (moabi), Entandrophragma cylindricum (sapelli) y Erythrophleum suaveolens (tali). Además, se buscaron interacciones con la fenología foliar y reproductiva y con el clima. En las tres especies, los anillos de crecimiento son anatómicamente distintos, anuales, y se forman al ritmo de las estaciones, con un incremento máximo durante la corta temporada de lluvias (de marzo a mayo). Las variaciones estacionales en el crecimiento se mostraron significativas en moabi y sapelli y no significativas en Tali. Los parámetros climáticos que tienen un papel determinante en dichas variaciones son la temperatura e insolación. El análisis dendrocronológico establece interesantes perspectivas para la adquisición de datos de crecimiento a largo plazo, necesarios para fijar los criterios de manejo de las poblaciones naturales de estas especies.

Palabras clave: Baillonella toxisperma, Entandrophragma cylindricum, Erythrophleum suaveolens, crecimiento, actividad cambial, fenología, estacionalidad de los anillos, bosque semicaduco, África Central. 


\section{Introduction}

La connaissance des rythmes de croissance en diamètre des arbres est indispensable à la compréhension de la dynamique des forêts tropicales (Jacoby, 1989). Elle s'avère cruciale aussi bien pour la gestion durable des peuplements forestiers (De Ridder et al., 2013) que pour la mise en œuvre du mécanisme de réduction des émissions dues à la déforestation et à la dégradation des forêts (REDD).

En Afrique centrale, la gestion durable repose notamment sur le calcul du taux de reconstitution pour chaque essence commerciale (Durrieu de Madron et Forni, 1997) et donc de leur vitesse de croissance diamétrique. Toutefois, les données actuellement disponibles proviennent d'un nombre de sites réduit et de périodes d'observation restreintes. Dès lors, toute généralisation demeure hasardeuse (Narboni, 2000 ; Picard et Gourlet-Fleury, 2008 ; Bourland et al., 2012). L'obtention de séquences de croissance des arbres sur le long terme est néanmoins possible par analyse des cernes (Brienen et Zuidema, 2006).

L'analyse des cernes nécessite cependant une prédisposition des arbres à produire des cernes visibles et anatomiquement distincts, ce qui n'est pas le cas de toutes les espèces ligneuses d'Afrique centrale (Groenendijk et al., 2014). Le caractère visible des cernes dans le bois dépendrait de particularités anatomiques propres à chaque espèce tandis que leur rythme de formation serait étroitement lié à la variation des conditions climatiques (Detienne et Mariaux, 1977 ; Worbes, 1995 ; Couralet et al., 2010).

Les rythmes phénologiques des arbres seraient surtout influencés par l'intensité des radiations solaires et l'ampleur de la saison sèche (Reich, 1995 ; Richardson et al., 2013). Les phases de feuillaison et de défeuillaison des arbres agissent sur l'activité photosynthétique, laquelle affecte la croissance primaire et secondaire, la survie et la productivité des arbres (Kariuki et al., 2006 ; Wagner et al., 2014). La phénologie foliaire et reproductive est selon Stahle et al. (1999) étroitement liée aux variations du climat local à l'échelle interannuelle et intra-annuelle, et pourrait s'avérer être un élément déterminant dans l'explication de la saisonnalité de la croissance diamétrique (Schöngart et al., 2002).

Ce type de relations demeure encore peu étudié en forêt tropicale (Schöngart et al., 2002 ; Cardoso et al., 2012 ; Morel et al., 2015). Il subsiste donc un manque crucial de données sur les réponses des forêts tropicales aux variations saisonnières du climat (Reed et al., 2012) et particulièrement dans le bassin du Congo.

La présente étude examine les relations entre la saisonnalité de la croissance diamétrique et les phénologies foliaire et reproductive de trois essences fortement exploitées en Afrique centrale pour la qualité de leur bois d'œuvre : Baillonella toxisperma Pierre (moabi, Sapotaceae), Entandrophragma cylindricum (Sprague) Sprague (sapelli, Meliaceae) et Erythrophleum suaveolens Guill. \& Perr. (tali, Fabaceae). Plus spécifiquement, il s'agit (1) de vérifier s'il est possible d'identifier distinctement les cernes de croissance dans le bois, et de déterminer quelles seraient les structures anatomiques qui les forment, (2) d'identifier quels seraient les rythmes de formation des cernes et leur lien avec les événements phénologiques du houppier, et (3) de préciser quelles seraient les variables climatiques impliquées.

\section{Matériel et méthodes}

\author{
Site d'étude
}

L'étude a été réalisée au sein des unités forestières d'aménagement (UFA) 10.030, 10.031, 10.039, 10.041, 10.042 et 10.044 gérées par la société forestière Pallisco, situées dans la région de l'Est du Cameroun (figure 1, A). Le site présente un paysage légèrement vallonné avec une altitude qui varie entre 600 et $760 \mathrm{~m}$. Les sols sont majoritairement de type ferralitique brun-rougeâtre et jaune (Jones et al., 2013), et reposent sur un socle géologique constitué de roches métamorphiques (Martin et al., 1966).

Le climat est équatorial de type guinéen, chaud et humide (Suchel, 1988), avec un régime pluviométrique bimodal (figure 1, B) caractérisé par une petite et une grande saison pluvieuse, respectivement PSP s'étalant de mars à juin et GSP d'août à novembre. Elles alternent avec une grande saison sèche (GSS, de novembre à mars) et une petite saison sèche (PSS en juillet-août). Celle-ci n'est toutefois pas une véritable saison sèche car elle correspond à une simple baisse des précipitations dont l'ampleur varie d'une année à l'autre. La température moyenne annuelle est relativement constante et reste inférieure à $25^{\circ} \mathrm{C}$. Les moyennes mensuelles les plus élevées sont enregistrées entre février et avril, et les plus faibles entre juillet et août. La courbe du rayonnement solaire présente également des maxima en février (GSS) puis en novembre (GSP), et des minima en janvier (GSS) puis en août (GSP).

Les forêts sont des forêts denses humides de transition entre les types sempervirent et semi-caducifolié (Fayolle et al., 2014). L'abondance d'espèces des familles de sterculiacées et ulmacées dans la canopée suggère toutefois une forte affinité avec le type semi-caducifolié (White, 1983 ; Letouzey, 1986).

\section{Espèces étudiées}

Les trois espèces étudiées représentent environ $30 \%$ de la production de grumes d'Afrique centrale et $23 \%$ de celle du Cameroun (De Wasseige et al., 2012).

$B$. toxisperma est un arbre caducifolié pouvant atteindre $60 \mathrm{~m}$ de hauteur et $3 \mathrm{~m}$ de diamètre. Il est hermaphrodite et produit des fruits (baies) qui sont consommés par de nombreux animaux (gorilles, éléphants...) et également exploités par les populations locales qui en extraient une huile alimentaire. Son aire de distribution va du Cameroun à la République du Congo. E. cylindricum est un arbre caducifolié pouvant atteindre $60 \mathrm{~m}$ de hauteur et dépasser $2 \mathrm{~m}$ de diamètre. Il est hermaphrodite et produit des capsules libérant des graines ailées dispersées par le vent. Son aire de distribution couvre toute la région guinéo-congolaise. Enfin, E. suaveolens est un arbre caducifolié atteignant $40 \mathrm{~m}$ de hauteur et $1,5 \mathrm{~m}$ de diamètre. Il est hermaphrodite et produit des gousses dont les graines sont principalement dispersées par autochorie. Son aire de distribution couvre toute la région guinéo-congolaise (Meunier et al., 2015). 


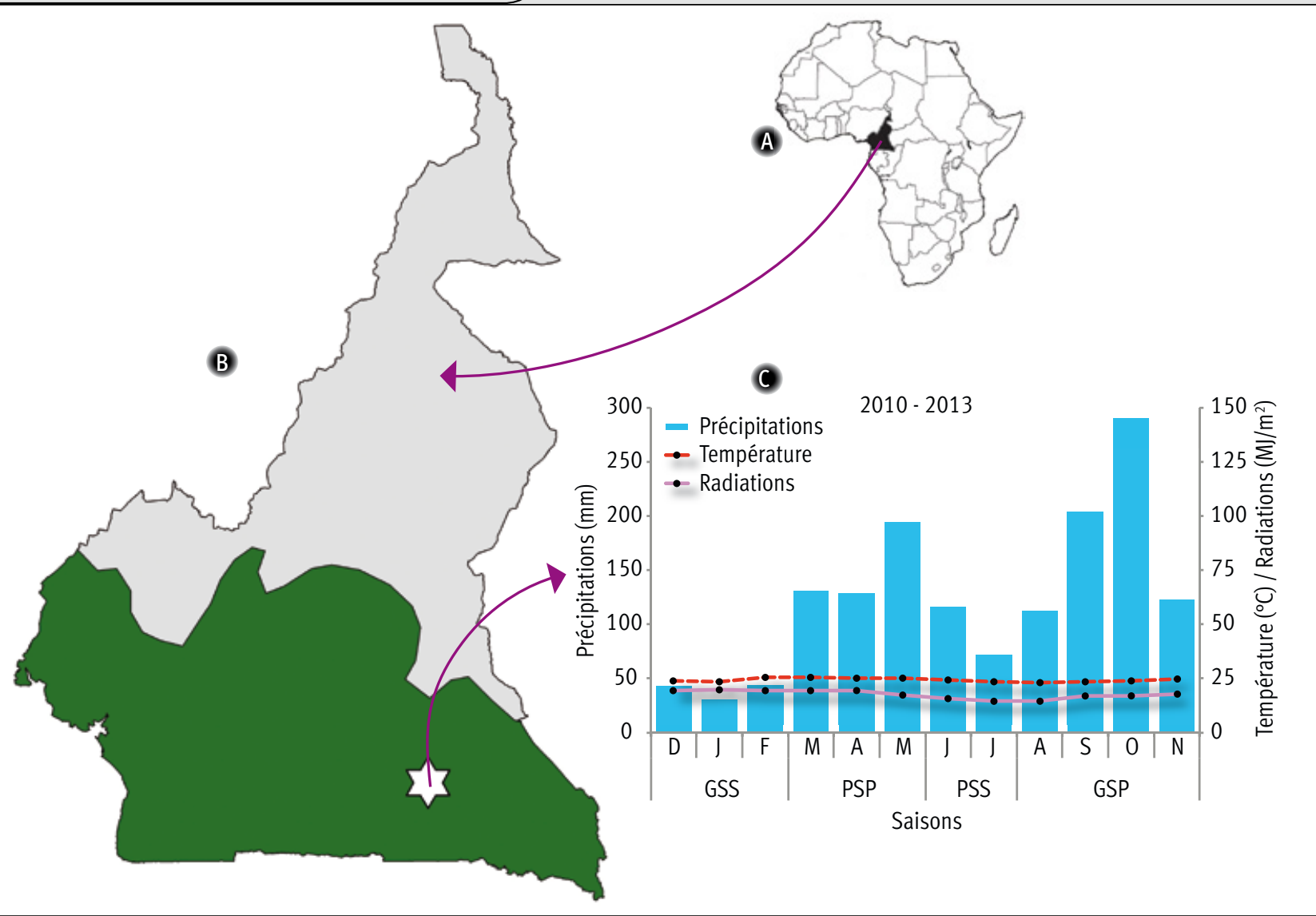

Figure 1.

Localisation du site d'étude en Afrique (A) et au Cameroun (B). La couleur verte représente la zone de forêt dense humide, et la grise, la zone de savane. Le diagramme climatique (C) de Lomié pour la période 2010-2013 est présenté à titre indicatif (les données de précipitations et de températures sont extraites du site de KNMI Explorer, et celles de radiations du site de la NASA Climatology Resource for Agroclimatology). GSP : grande saison pluvieuse ; GSS : grande saison sèche ; PSP : petite saison pluvieuse ; PSS : petite saison sèche.

La nature et la périodicité des cernes de croissance ont été abordées pour $E$. cylindricum et $E$. suaveolens en Afrique centrale et de l'Ouest (Detienne et al., 1998), notamment au Cameroun (Nzogang, 2009 ; Groenendijk et al., 2014). Par contre, B. toxisperma a fait l'objet de peu d'études anatomiques (Detienne et al., 1998 ; Durrieu de Madron et al., 2000). La croissance annuelle diamétrique des trois espèces varie de 0,72 à $0,95 \mathrm{~cm} /$ an pour $B$. toxisperma, de 0,47 à $0,64 \mathrm{~cm} /$ an pour $E$. cylindricum, et de 0,45 à $0,68 \mathrm{~cm} / \mathrm{an}$ pour E. suaveolens (Durrieu de Madron et al., 2000 ; Fétéké et al., 2015). Quelques travaux se sont intéressé à leur phénologie foliaire et reproductive (Bibani Mbarga et al., 1999 ; Durrieu de Madron et Daumerie, 2004). Les arbres ont tendance à être défeuillés pendant la période la plus sèche (entre décembre et janvier), fleurissent en fin de saison sèche (février) et fructifient dès l'apparition des premières pluies (fructification et dissémination entre mars et octobre).

\section{Suivi de la croissance}

L'analyse de la saisonnalité et de la variabilité de la croissance diamétrique a été réalisée par échantillonnage destructif de 12 arbres : deux de $B$. toxisperma, cinq de $E$. cylindricum et cinq de $E$. suaveolens. Le diamètre initial des arbres a été mesuré et leur statut classé en deux catégories en fonction de la position du houppier dans la canopée : dominant ou codominant.

La saisonnalité de la croissance a été étudiée selon la technique du marquage du cambium préconisée par Mariaux (1967). Le marquage du cambium a été réalisé de façon séquentielle, au rythme de quatre fois par an pendant trois années (août 2010 à juin 2013). Lors des changements de saison (en mars pour la PSP, juin pour la PSS, août pour la GSP et novembre pour la GSS), une entaille a été pratiquée à la même hauteur en suivant un sens unique sur la circonférence de l'arbre, à l'aide d'un ciseau à bois enfoncé directement dans l'écorce jusqu'au cambium de façon à laisser une marque. Chaque arbre a ainsi reçu huit entailles permettant d'apprécier le rythme de formation de trois couches annuelles successives de cernes.

Des échantillons de bois de $15 \times 10 \times 5 \mathrm{~cm}$ pourvus d'écorce ont été prélevés en juin 2013 à la tronçonneuse au niveau des entailles sur chaque arbre abattu. Au total, ont pu être collectés 14 échantillons de B. toxisperma, 39 de $E$. cylindricum et 37 de $E$. suaveolens. Ils ont été archivés et analysés dans le laboratoire du Service de biologie du bois du Musée royal de l'Afrique centrale (MRAC, Tervuren, Belgique). 
L’identification anatomique des cernes a été réalisée par observation au microscope photonique (Olympus BX60) de coupes fines (16 à $18 \mu \mathrm{m}$ ) de la section transversale des échantillons de bois collectés. Les coupes ont été effectuées au microtome électronique (Microm HM 440E) sur des cubes de $1 \mathrm{~cm}$ de côté, contenant du bois et de l'écorce. Pour chaque échantillon, la coloration et le montage des coupes ont été réalisés selon le protocole décrit par Dié et al. (2012).

L'annualité et la largeur des cernes ont été déterminées à partir d'images numériques à très haute résolution (1 200 et 3500 dpi) des sections transversales poncées des échantillons. Pour chaque échantillon, les cicatrices ainsi que les cernes nouvellement formés ont été identifiés sur l'image numérique générée, et datés, puis digitalisés avec le logiciel ArcGis (Latte et al., 2014). Les cernes sont considérés comme annuels si le nombre de cernes identifiés entre la cicatrice et l'écorce correspond exactement au nombre d'années qu'a duré l'expérimentation (Stahle et al., 1999). La position de chaque cicatrice dans le cerne détermine le niveau de formation de ce dernier au fil des saisons. Pour chaque échantillon, les mesures de la portion de largeur de cerne précédant chaque cicatrice (largeur saisonnière $L s$, en $\mathrm{mm}$ ), ainsi que de la largeur totale du cerne formé (largeur annuelle $L t$, en $\mathrm{mm}$ ), ont été effectuées en dehors de la zone d’influence des cicatrices.

\section{Suivi des phénologies foliaire et reproductive}

La phénologie des trois espèces étudiées a été suivie mensuellement sur le site d'étude pendant 36 mois (juillet 2010 à août 2013). L'effectif consistait en 20 arbres de B. toxisperma, 70 de E. cylindricum et 60 de E. suaveolens de diamètre compris entre 60 et $158 \mathrm{~cm}$. Le suivi a été réalisé par observation directe du houppier des arbres. La quantification de ces phases phénologiques (défeuillaison, feuillaison, floraison, fructification immature et fructification mature) a été réalisée par estimation de la proportion du houppier couverte par les différents organes (Morellato et al., 2010 ; Bourland et al., 2012).

\section{Données climatiques}

Les données journalières de précipitations et de température ont été extraites du site web de KNMI Explorer ${ }^{1}$ et celles de rayonnement solaire extraites du site de la «Climatology Resource for Agroclimatology $»^{2}$ de la NASA. Il s'agit de totaux (précipitations en $\mathrm{mm}$ ) ou de moyennes (température en ${ }^{\circ} \mathrm{C}$ et radiations en $\mathrm{MJ} / \mathrm{m}^{2}$ ) correspondant à la période 2010-2013 pour la zone d'étude (données des stations météorologiques de Lomié, Abong Mbang et Yokadouma).

\section{Analyse des données}

La croissance périodique (saisonnière et annuelle) a été déterminée par mesure des largeurs de cernes relevées au niveau des marques faites sur le cambium pour chaque

\footnotetext{
${ }^{1}$ http://climexp.knmi.nl/

2 http://power.larc.nasa.gov/
}

échantillon. Les accroissements annuels ( $A c c_{0}$, en mm/an) et moyens périodiques $\left(A c c_{m}\right.$ en $\left.\mathrm{mm} / \mathrm{an}\right)$ ainsi que les accroissements saisonniers $\left(A c c_{s}\right.$ en $\left.\mathrm{mm}\right)$ et relatifs $\left(A c c_{r}\right.$ en \%) ont été calculés pour chaque arbre et pour chaque espèce.

Pour analyser la phénologie, le taux de recouvrement des arbres par les feuilles (RFol), les nouvelles feuilles uniquement (RFeu), les fleurs (RFlo), les fruits immatures (RFri) et matures $(R F r m)$ ont préalablement été déterminés par mois d'observation. Le cycle des événements phénologiques pour chaque espèce a ensuite été déterminé par analyse graphique de la distribution des taux de recouvrement de la cime des arbres par les différents organes en fonction du temps (Newstrom et al., 1994), complétée par une analyse statistique circulaire permettant de tester le degré d'agrégation temporel des événements phénologiques (Davies et Ashton, 1999). Un test d'uniformité de Rayleigh (Davies et Ashton, 1999) a été réalisé pour vérifier la significativité de la distribution des événements phénologiques des espèces.

Pour caractériser le climat de la zone, les moyennes de précipitations (Pmoy), de radiation (Rmoy) et de température (Tmoy) ont été calculées pour chaque saison. Les saisons climatiques ont été considérées comme une succession de mois pluvieux ou secs, indépendamment de l'année calendaire. Ainsi, la grande saison sèche va de décembre de l'année $A_{o}$ à février de l'année $A_{1}$ et l'année couvre les mois de décembre $A_{0}$ à novembre $A_{1}$. Les coefficients de variation ont été utilisés pour décrire la variabilité intra-annuelle du climat.

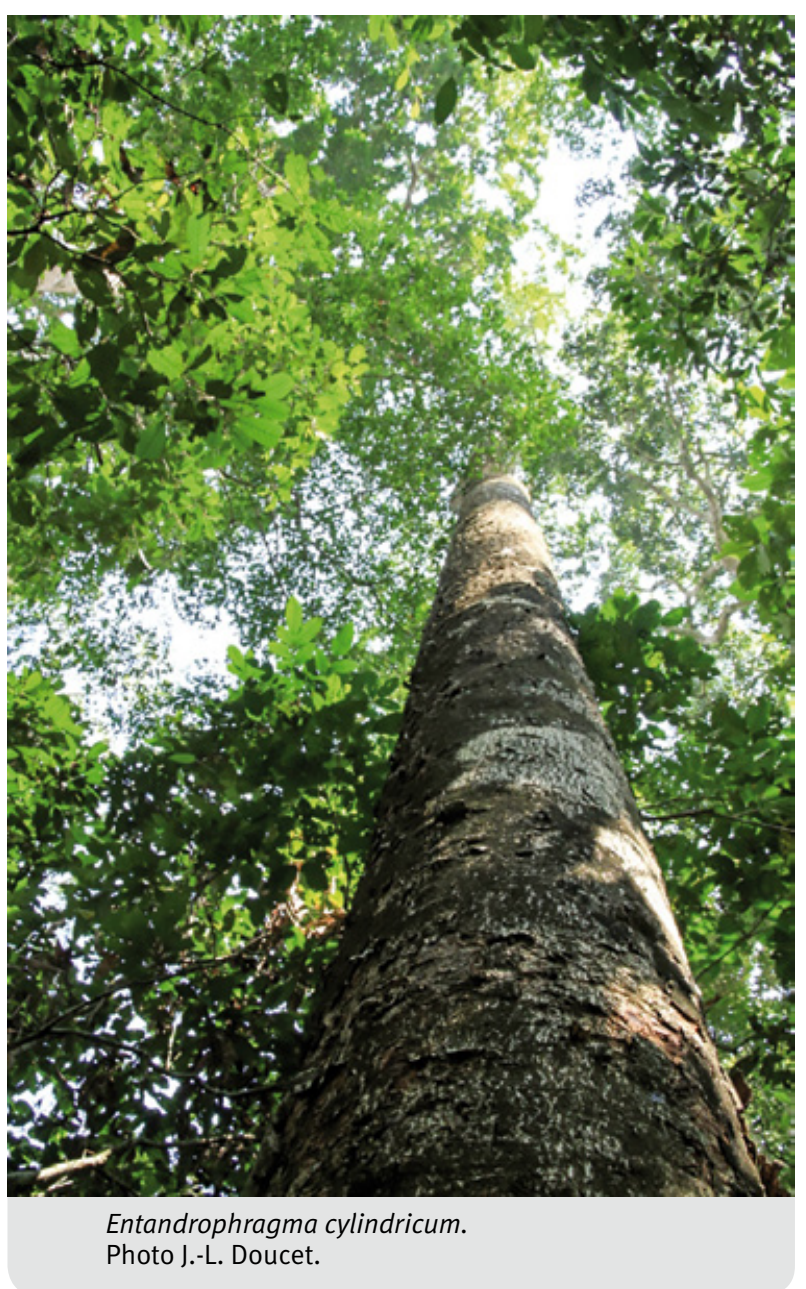




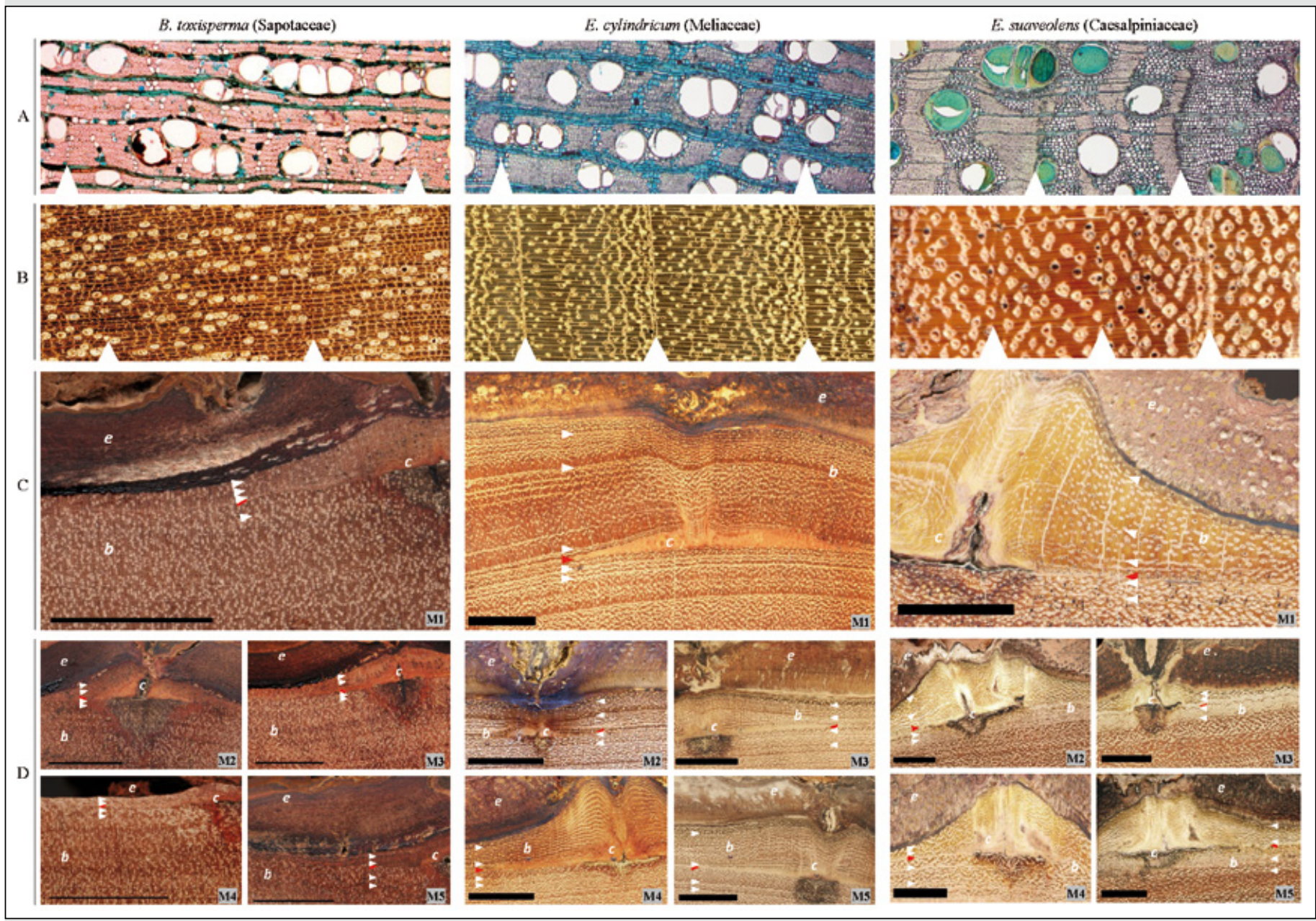

Figure 2.

Profils microscopique et macroscopique de la section transversale poncée des échantillons de bois montrant les limites des cernes annuels (triangles blancs) (A et B respectivement), les nouvelles couches de bois contenant la cicatrice (triangle rouge) résultant du marquage cambial (C et D). M1 correspond au marquage initial datant de trois ans, M2 à M5 correspondent aux quatre marquages saisonniers réalisés en fin de grande saison sèche, de petite saison pluvieuse, de petite saison sèche et de grande saison pluvieuse respectivement. La bande noire présente sur chaque image correspond à l'échelle de $1 \mathrm{~cm}$. $e$ : écorce ; $b:$ bois ; $c:$ cicatrice.

\section{Résultats}

\section{Anatomie des cernes}

Enfin, pour explorer les facteurs explicatifs potentiels des variations de la croissance, une régression linéaire multiple a été ajustée selon la méthode pas à pas ascendante à partir des données climatiques et phénologiques pour identifier les variables les plus corrélées à l'accroissement saisonnier $(A C C)$ de chacune des trois espèces.

Le degré d'agrégation temporel des événements phénologiques a été testé à l'aide du package «CircStats » (Jammalamadaka et SenGupta, 2001) du logiciel R (R Development Core Team, 2013). La significativité des corrélations a été appréciée par le biais des coefficients de détermination ajustés ( $\left.R^{2} a d j\right)$ et des probabilités $(P)$.
L'examen des coupes et des sections transversales a montré que les cernes sont anatomiquement visibles, mais la limite entre ces derniers est plus ou moins facilement distinguable selon l'espèce (figure $2, A$ et $B$ et annexe 1 ). Le cerne se présente globalement comme une singularité locale tangentielle d'un ou plusieurs tissus constitutifs de la matrice, disposés et délimités différemment selon les espèces.

Chez B. toxisperma, la limite entre cernes est marquée par une ligne discontinue de cellules de parenchyme disposées en chaînette et associées à peu de vaisseaux souvent de plus petite taille. Elle est précédée d'une couche de fibres constituée de trois à cinq rangées de cellules aplaties radialement et à paroi épaissie, et suivie d'une couche de parenchyme dilaté en réseau (annexe 1, A). Chez E. cylindricum, la 
limite du cerne correspond à une bande continue de parenchyme marginal (trois à quatre rangées de cellules) associée à très peu de vaisseaux de petite taille (annexe $1, B$ ). Chez E. suaveolens, la limite du cerne est moins distincte que pour B. toxisperma et $E$. cylindricum, et correspond à une bande discontinue de parenchyme formant de courtes ailes autour des vaisseaux de petite taille (annexe 1, C).

\section{Annualité des cernes de croissance}

Les cernes des trois espèces sont annuels, les plans ligneux des échantillons poncés révélant la présence de trois nouvelles couches qui recouvrent la cicatrice (M1) qui date de trois ans (figure 2, C et D). Les cernes annuels se forment en grande partie pendant la période pluvieuse (mars à novembre), les cicatrices issues des marquages de mars (M2) et de novembre (M5) étant respectivement proches de la limite entre le cerne précédent et celui en formation, respectivement (figure 2, D). Les cicatrices des marquages de juin (M3) et d'août (M4) sont quant à elles situées toutes les deux à différents niveaux dans la couche de cerne en formation. La grande saison sèche (décembre à février) semble correspondre à une période de croissance ralentie pendant laquelle les arbres achèvent la production du bois final du cerne précédent et amorcent celle du bois initial du cerne suivant.

\section{Variabilité de la croissance}

L'accroissement moyen périodique $\left(A c c_{m}\right)$ varie entre arbres étudiés de 1,75 à $2,24 \mathrm{~mm}$ pour $B$. toxisperma, de 3,03 à $6,68 \mathrm{~mm}$ pour $E$. cylindricum et de 3,22 à $6,37 \mathrm{~mm}$ pour E. suaveolens (tableau I). L'accroissement annuel (Acc) varie également entre années, avec une légère tendance à la diminution pour $B$. toxisperma et plutôt à une augmentation pour $E$. cylindricum et $E$. suaveolens.

\section{Tableau I.}

Accroissement annuel ( $A c c_{a}$, en mm/an) et accroissement moyen périodique ( $A c c_{m}$, en mm/an) des arbres mesurés sur la période 2010-2012 pour Baillonella toxisperma Pierre, Entandrophragma cylindricum (Sprague) Sprague et Erythrophleum suaveolens Guill. \& Perr. Le statut social de chaque arbre, son diamètre à hauteur de poitrine $(D h p$, en $\mathrm{cm})$, la hauteur de marquage $(H$, en $\mathrm{cm})$, les nombres de marquages reçus $(M)$, d'échantillons de bois prélevés $(E)$ et de mesures de largeur de cernes réalisées $(M e)$ sont précisés par arbre.

\begin{tabular}{|c|c|c|c|c|c|c|c|c|c|c|}
\hline Arbres & Statut social & Dhp & $\boldsymbol{H}$ & $M$ & $\boldsymbol{E}$ & Me & 2010 & $\begin{array}{l}A c c_{a} \\
2011\end{array}$ & 2012 & $\begin{array}{c}A C c_{m} \\
2010-2012\end{array}$ \\
\hline \multicolumn{11}{|c|}{ Moabi (Baillonella toxisperma Pierre) } \\
\hline Tw64567 & Dominant & 140 & 320 & 7 & 7 & 27 & $2,92 \pm 0,39$ & $2,14 \pm 0,4$ & $1,66 \pm 0,56$ & $2,24 \pm 0,45$ \\
\hline Tw64568 & Codominant & 170 & 265 & 7 & 7 & 24 & $2,04 \pm 0,26$ & $1,4 \pm 0,3$ & $1,82 \pm 0,33$ & $1,75 \pm 0,30$ \\
\hline Moyenne & & & & & & & $2,48 \pm 0,32$ & $1,77 \pm 0,35$ & $1,74 \pm 0,45$ & $2,00 \pm 0,37$ \\
\hline \multicolumn{11}{|c|}{ Sapelli (Entandrophragma cylindricum (Sprague) Sprague) } \\
\hline Tw64570 & Dominant & 123 & 145 & 8 & 8 & 19 & $6,09 \pm 1,6$ & $2,99 \pm 1,24$ & $2,92 \pm 1,12$ & $5,84 \pm 0,6$ \\
\hline Tw64572 & Dominant & 100 & 142 & 8 & 7 & 19 & $4,67 \pm 0,93$ & $3,8 \pm 1,12$ & $0,85 \pm 0,21$ & $4,00 \pm 1,32$ \\
\hline Tw64569 & Codominant & 121 & 154 & 8 & 8 & 28 & $4,00 \pm 0,30$ & $4,79 \pm 1,06$ & $8,75 \pm 0,46$ & $6,68 \pm 0,80$ \\
\hline Tw64571 & Codominant & 104 & 150 & 8 & 8 & 29 & $4,30 \pm 1,44$ & $5,52 \pm 0,44$ & $10,22 \pm 0,52$ & $3,11 \pm 0,75$ \\
\hline Tw64573 & Codominant & 120 & 172 & 8 & 8 & 22 & $4,68 \pm 0,71$ & $3,29 \pm 0,66$ & $1,13 \pm 0,28$ & $3,03 \pm 0,55$ \\
\hline Moyenne & & & & & & & $4,75 \pm 1,00$ & $4,08 \pm 0,90$ & $4,77 \pm 0,52$ & $4,53 \pm 0,81$ \\
\hline \multicolumn{11}{|c|}{ Tali (Erythrophleum suaveolens Guill. \& Perr.) } \\
\hline Tw64574 & Dominant & 95 & 155 & 8 & 8 & 14 & $3,33 \pm 0,98$ & $3,96 \pm 1,14$ & $6,29 \pm 2,97$ & $4,53 \pm 1,69$ \\
\hline Tw64575 & Dominant & 111 & 123 & 8 & 6 & 15 & $1,16 \pm 0,60$ & $3,95 \pm 0,82$ & $6,52 \pm 4,29$ & $3,88 \pm 1,90$ \\
\hline Tw64577 & Dominant & 109 & 130 & 8 & 8 & 17 & $3,17 \pm 0,37$ & $3,00 \pm 0,45$ & $3,47 \pm 1,84$ & $4,10 \pm 1,09$ \\
\hline Tw64576 & Codominant & 88 & 120 & 8 & 8 & 11 & 1 & $4,00 \pm 0,89$ & $4,19 \pm 1,29$ & $3,22 \pm 0,88$ \\
\hline Tw64578 & Codominant & 100 & 149 & 8 & 7 & 10 & $3,96 \pm 0,88$ & $8,77 \pm 3,00$ & 1 & $6,37 \pm 1,94$ \\
\hline Moyenne & & & & & & & $2,90 \pm 0,71$ & $4,74 \pm 1,26$ & $5,12 \pm 2,6$ & $4,42 \pm 1,50$ \\
\hline
\end{tabular}

/ : Valeur manquante.

La hauteur de marquage plus élevée fixée pour les deux arbres de moabi se justifie par la présence de hauts contreforts à la base du tronc des arbres âgés (diamètre 140 à $170 \mathrm{~cm}$ ), un trait caractéristique de l'espèce. 


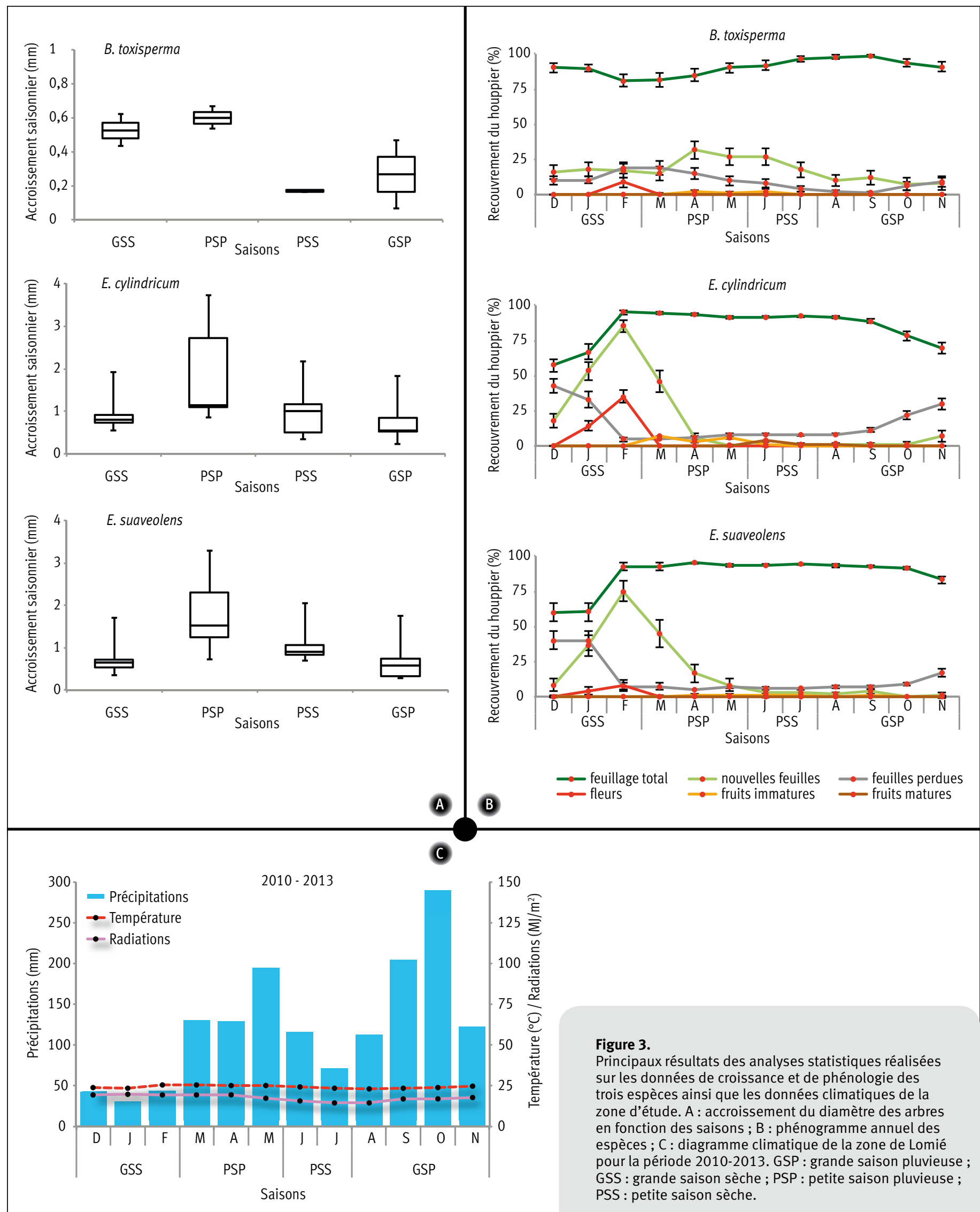


Tableau II.

Accroissement saisonnier ( $A c c_{s}$, en $\left.\mathrm{mm}\right)$ et accroissement relatif cumulé $(A c c$, en \%) mesurés pour Baillonella toxisperma Pierre, Entandrophragma cylindricum (Sprague) Sprague et Erythrophleum suaveolens Guill. \& Perr. sur la période 2010-2012. GSS : grande saison sèche ; PSP : petite saison pluvieuse ; PSS : petite saison sèche ; PSP : petite saison pluvieuse. Le statut social ainsi que l'accroissement annuel périodique $\left(A c c_{m}\right.$, en $\left.\mathrm{mm}\right)$ sont également indiqués pour chaque arbre.

\begin{tabular}{|c|c|c|c|c|c|c|c|c|c|c|}
\hline \multirow[t]{2}{*}{ Arbres } & \multirow[t]{2}{*}{ Statut social } & \multirow{2}{*}{$A C c_{m}$} & \multicolumn{2}{|c|}{ GSS } & \multicolumn{2}{|c|}{ PSP } & \multicolumn{2}{|c|}{ PSS } & \multicolumn{2}{|c|}{ GSP } \\
\hline & & & $\operatorname{Acc}_{\mathrm{s}}$ & $A c c_{r}$ & $\operatorname{Acc}_{\mathrm{s}}$ & $A_{C C}$ & $\operatorname{Acc}_{\mathrm{s}}$ & $A c c_{r}$ & $\operatorname{Acc}_{\mathrm{s}}$ & $A c c_{r}$ \\
\hline \multicolumn{11}{|c|}{ Moabi (Baillonella toxisperma Pierre) } \\
\hline Tw64567 & Dominant & $2,24 \pm 0,45$ & $0,62 \pm 0,36$ & 27,7 & $0,53 \pm 0,13$ & 51,5 & $0,17 \pm 0,03$ & 59,1 & $0,47 \pm 0,09$ & 80,1 \\
\hline Tw64568 & Codominant & $1,75 \pm 0,30$ & $0,43 \pm 0,23$ & 24,7 & $0,66 \pm 0,04$ & 62,6 & l & l & $0,06 \pm 0,23$ & 75,6 \\
\hline Moyenne & & $2,00 \pm 0,31$ & $0,53 \pm 0,24$ & 26,2 & $0,60 \pm 0,06$ & 57,1 & $0,17 \pm 0,03$ & 59,1 & $0,27 \pm 0,06$ & 77,8 \\
\hline
\end{tabular}

Sapelli (Entandrophragma cylindricum (Sprague) Sprague)

\begin{tabular}{|c|c|c|c|c|c|c|c|c|c|c|}
\hline Tw64570 & Dominant & $4,00 \pm 1,32$ & $1,04 \pm 1,24$ & 26,1 & $2,73 \pm 0,21$ & 55,0 & $1,17 \pm 0,12$ & 88,1 & $0,84 \pm 0,09$ & 93,9 \\
\hline Tw64572 & Dominant & $3,11 \pm 0,75$ & $0,92 \pm 0,70$ & 29,7 & $1,16 \pm 0,51$ & 64,8 & $1,33 \pm 0,44$ & 75,9 & $0,23 \pm 0,08$ & 93,0 \\
\hline w64569 & Codominant & $5,84 \pm 0,60$ & $0,55 \pm 0,55$ & 9,4 & $2,72 \pm 0,30$ & 56,1 & $1,01 \pm 0,12$ & 76,1 & $1,54 \pm 0,18$ & 0, \\
\hline Tw64571 & Codominant & $6,68 \pm 0,80$ & $0,73 \pm 0,72$ & 11,0 & $1,09 \pm 0,21$ & 51,8 & $0,34 \pm 0,08$ & 66,9 & $0,53 \pm 0,13$ & 9, \\
\hline w64573 & Codominant & $3,03 \pm 0,55$ & $0,81 \pm 0,49$ & 26,7 & $0,86 \pm 0,19$ & 55,0 & $0,51 \pm 0,09$ & 71,7 & $0,53 \pm 0,10$ & 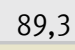 \\
\hline Moyenne & & $4,53 \pm 0,95$ & $0,93 \pm 0,87$ & 20,6 & $1,63 \pm 0,33$ & 56,5 & $0,87 \pm 0,18$ & 75,7 & $0,71 \pm 0,15$ & 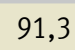 \\
\hline
\end{tabular}

Tali (Erythrophleum suaveolens Guill. \& Perr.)

\begin{tabular}{|c|c|c|c|c|c|c|c|c|c|c|}
\hline Tw64574 & Dominant & $4,53 \pm 1,69$ & $0,66 \pm 1,59$ & 14,6 & $2,30 \pm-0,48$ & 65,3 & $0,69 \pm 0,26$ & 80,6 & $0,59 \pm 0,22$ & 93,7 \\
\hline Tw64575 & Dominant & $3,88 \pm 1,90$ & $0,53 \pm 0,00$ & 13,6 & $1,52 \pm 1,01$ & 52,9 & $0,84 \pm 0,41$ & 74,5 & I & 1 \\
\hline Tw64577 & Dominant & $3,22 \pm 0,88$ & $0,71 \pm 0,77$ & 22,2 & $1,24 \pm-0,33$ & 44,8 & $0,91 \pm 0,24$ & 77,7 & $0,32 \pm 0,09$ & 86,5 \\
\hline Tw64576 & Codominant & $4,10 \pm 1,09$ & $1,02 \pm 0,94$ & 24,9 & $0,73 \pm-0,37$ & 55,3 & $1,06 \pm 0,29$ & 77,6 & $0,28 \pm 0,08$ & 85,5 \\
\hline Tw64578 & Codominant & $6,37 \pm 1,94$ & $0,35 \pm 1,80$ & 5,60 & I & 1 & $3,59 \pm 1,10$ & 56,4 & $2,31 \pm 0,71$ & 92,7 \\
\hline Moyenne & & $4,42 \pm 0,96$ & $0,71 \pm 0,86$ & 16,2 & $1,7 \pm-0,34$ & 54,6 & $0,83 \pm 0,18$ & 73,4 & $0,72 \pm 0,16$ & 89,6 \\
\hline
\end{tabular}

/ : Valeur manquante.

Les marquages réalisés lors des changements de saison n'ont pas permis d'obtenir la valeur de $100 \%$ de l'accroissement relatif cumulé sur l'année parce que la formation du bois final du cerne précédent et celle du bois initial du cerne suivant s'effectuent au cours d'une même saison (la grande saison sèche pour les trois espèces étudiées).

Les mesures de largeurs saisonnières des cernes montrent que la croissance est continue mais variable en fonction des saisons, suggérant ainsi l'absence de période de dormance pour ces trois espèces (figure 3, A). La croissance des arbres en grande saison sèche ne dépasse toutefois pas $26 \%$ de l'accroissement annuel (tableau II). La croissance la plus importante est obtenue pendant la petite saison pluvieuse (30 à $45 \%$ de l'accroissement annuel selon l'espèce).

\section{Phénologies foliaire et reproductive des espèces}

Le cycle phénologique des espèces étudiées est de type annuel, calqué surtout sur les saisons (figure 3, B). Les différents événements phénologiques ont lieu une seule fois dans l'année avec des épisodes relativement courts (trois à cinq semaines) pour la défeuillaison ou la floraison, relativement longs pour la feuillaison ou la fructification (immature et mature).

$B$. toxisperma et E. cylindricum présentent un comportement phénologique plutôt similaire. L'activité phénologique est globalement synchrone pour les 20 et 60 arbres respectivement suivis ( $r$ rosp $_{\text {esp }}$ proche de 1, tableau III), à l'exception de la feuillaison chez $B$. toxisperma et de la défeuillaison chez $E$. cylindricum qui varient en fonction des individus ( $r h o_{\text {esp }}$ et $r h o_{\text {ind }}$ faibles). La période de plein feuillage (taux de recouvrement supérieur à $90 \%$ ) couvre huit mois (mai à décembre pour $B$. toxisperma et février à octobre pour E. cylindricum). Les phases de floraison et de fructification (immature et mature) sont annuelles et concentrées dans le temps même si, pour B. toxisperma, les arbres montrent individuellement un épisode de fructification relativement long 
$\left(r h o_{\text {ind }}<0,5\right)$. Les pics de défeuillaison et de floraison sont simultanés et observés en février, à la fin de la grande saison sèche. La dissémination des fruits survient pendant la petite saison sèche (juillet), après trois mois de maturation.

Pour $E$. suaveolens, les événements phénologiques sont plus étalés dans le temps et variables selon les individus ( $r h o_{\text {esp }}$ et $r h o_{\text {ind }}$ faibles), à l'exception de la floraison qui est très brève $\left(r h o_{\text {esp }}\right.$ et $\left.r h o_{i n d}>0,5\right)$. La défeuillaison et la feuillaison s'étalent sur plusieurs mois, le pic étant observé en grande saison sèche (janvier et février respectivement). La floraison intervient en fin de grande saison sèche (pic en février), la fructification commence en petite saison sèche (juillet), avec un pic en grande saison pluvieuse (octobre). La dissémination des graines intervient après cinq à sept mois.

\section{Relation entre le climat, la croissance et la phénologie des espèces}

Le climat de la zone d'étude a faiblement varié sur la période allant de 2010 à 2013 (annexe 2). Les variations annuelles des moyennes de précipitations, de température et de radiation sont très faibles ( $c v<5 \%)$. Les moyennes saisonnières de température et de radiation varient également faiblement ( $c v<11 \%)$ alors que celles de précipitations varient fortement ( $c v=53 \%)$. L'analyse de corrélation linéaire réalisée entre les moyennes saisonnières et les données de l'accroissement saisonnier $\left(A c c_{s}\right)$, ainsi que de la phénologie de chacune des trois espèces, montre que le climat est significativement corrélé avec la croissance du diamètre des arbres.

\section{Tableau III.}

Résultats récapitulant les principales variables descriptives de l'analyse circulaire ainsi que les statistiques de base du test de l'uniformité de la distribution de Rayleigh, appliquée aux données phénologiques des trois espèces étudiées dans une forêt semi-caducifoliée du sud-est du Cameroun. $n$ : nombre d'observations ; mean.dir: direction moyenne du vecteur; $r$ o ${ }_{\text {ind }}$ : longueur moyenne du vecteur à l'échelle de chaque arbre ; rhoesp : longueur moyenne du vecteur à l'échelle de tous les arbres ; $p$-value : significativité du test de Rayleigh ; GSP : grande saison pluvieuse ; GSS : grande saison sèche ; PSP : petite saison pluvieuse ;

PSS : petite saison sèche.

Espèces

Moabi

(Baillonella toxisperma Pierre)

\section{Sapelli}

(Entandrophragma cylindricum

(Sprague)

Sprague)

Tali

(Erythrophleum suaveolens Guill. \& Perr.)

\section{Statistiques}

rho
rhd
rho
esp

mean.dir

p-value

Date du pic moyen

Saison correspondante

Défeuillaison

(

Période : juillet 2010 - août 2013

Feuillaison Floraison

\section{Fructification} immature

\section{Fructification} mature

\begin{tabular}{|c|c|c|c|c|c|}
\hline$n$ & 202 & 137 & 26 & 69 & 19 \\
\hline$r_{\text {ind }}$ & 0,987 & 0,278 & 0,995 & 0,498 & 0,302 \\
\hline rho $_{e s p}$ & 0,595 & 0,456 & 0,986 & 0,864 & 0,953 \\
\hline mean.dir & 0,592 & 2,367 & 1,106 & 2,508 & $-2,841$ \\
\hline$p$-value & 0,038 & 0,820 & 0,035 & 0,517 & 0,791 \\
\hline Date du pic moyen & Février & Mai & Février & Mai & Juillet \\
\hline Saison correspondante & GSS & PSP & GSS & PSP & PSS \\
\hline$n$ & 2042 & 523 & 194 & 241 & 130 \\
\hline$r_{\text {ind }}$ & 0,392 & 0,935 & 0,705 & 0,784 & 0,285 \\
\hline$r h o_{e s p}$ & 0,151 & 0,637 & 0,966 & 0,821 & 0,912 \\
\hline mean.dir & $-2,631$ & 0,870 & 0,881 & 2,277 & $-2,750$ \\
\hline$p$-value & 0,670 & 0,059 & 0,244 & 0,164 & 0,811 \\
\hline Date du pic moyen & Janvier & Février & Février & Mai & Juillet \\
\hline Saison correspondante & GSS & GSS & GSS & PSP & PSS \\
\hline
\end{tabular}

\begin{tabular}{|c|c|c|c|c|c|}
\hline$n$ & 1342 & 399 & 49 & 95 & 29 \\
\hline$r h o_{\text {ind }}$ & 0,395 & 0,462 & 0,559 & 0,359 & 0,429 \\
\hline$r h o_{\text {esp }}$ & 0,169 & 0,430 & 0,909 & 0,590 & 0,409 \\
\hline mean.dir & $-2,410$ & 1,401 & 0,858 & $-2,764$ & $-1,495$ \\
\hline$p$-value & 0,666 & 0,569 & 0,430 & 0,717 & 0,618 \\
\hline Date du pic moyen & Janvier & Février & Février & Juillet & Octobre \\
\hline Saison correspondante & GSS & GSS & GSS & PSS & GSP \\
\hline
\end{tabular}


Généralement, l'intensité du recouvrement foliaire est corrélée positivement aux précipitations, à l'inverse du renouvellement des feuilles et de la floraison. Le rayonnement moyen est corrélé négativement avec le recouvrement foliaire et la fructification mais positivement avec la floraison et l'apparition des nouvelles feuilles. La croissance est également corrélée avec le climat. La température moyenne est corrélée positivement à la croissance des trois espèces, de même que le rayonnement moyen chez $B$. toxisperma.

Les corrélations de la croissance radiale avec les phénophases sont davantage variables selon les espèces. Le recouvrement foliaire est corrélé positivement à la croissance de $E$. cylindricum mais négativement à celle de B. toxisperma. Il n'y a aucune corrélation avec la floraison et une influence de la fructification n'est perceptible que pour $E$. cylindriucm. La sélection des variables par régression multiple utilisant la méthode pas à pas ascendante fait ressortir le recouvrement foliaire pour $B$. toxisperma, le recouvrement en fruits immatures pour $E$. cylindricum et la température pour $E$. suaveolens comme principaux facteurs explicatifs des variations saisonnières de l'accroissement des trois espèces $\left(R^{2} a d j=0,605,0,606\right.$ et 0,273 respectivement). La valeur des précipitations contribue également à améliorer de $24 \%$ la proportion de variance expliquée par le couvrement foliaire dans le seul cas de $B$. toxisperma.

\section{Discussion}

La nature et le rythme de formation des cernes de croissance ainsi que le comportement phénologique de trois essences ( $B$. toxisperma, $E$. cylindricum et $E$. suaveolens) en réponse aux variations saisonnières du climat ont été examinés dans le sud-est du Cameroun. L'étude a démontré que les trois essences forment des cernes annuels anatomiquement distincts et que leur croissance est continue sur les saisons, avec toutefois un ralentissement pendant la grande saison sèche. Le cycle phénologique de ces espèces est également annuel avec une phase végétative marquée en grande saison sèche et une phase reproductive en saison pluvieuse.

\section{Variabilité anatomique et rythme de formation des cernes de croissance}

Les caractéristiques anatomiques des trois espèces étudiées laissent transparaître des unités structurelles régulièrement délimitées par une ligne de parenchyme et/ou une couche de fibres à paroi plus épaisse et de diamètre radial réduit (chez $B$. toxisperma), correspondant à des limites entre des cernes (figure 2). Ces particularités anatomiques constituent quelques-unes des principales caractéristiques retenues par plusieurs auteurs pour identifier formellement la limite des cernes dans certains bois tropicaux (Mariaux, 1967 ; Detienne et al., 1998 ; Trouet et al., 2001 ; Worbes et Fichtler, 2010). L'identification macroscopique sur les sections poncées et microscopique sur les coupes fines des sections transversales des échantillons de bois (figures 2, A et B) confirme bien l'existence et la lisibilité des cernes dans le bois des trois espèces étudiées. La couche de parenchyme marginal délimitant les cernes, continue chez $E$. cylindricum et plutôt discontinue chez $B$. toxisperma et $E$. suaveolens, confère une visibilité très

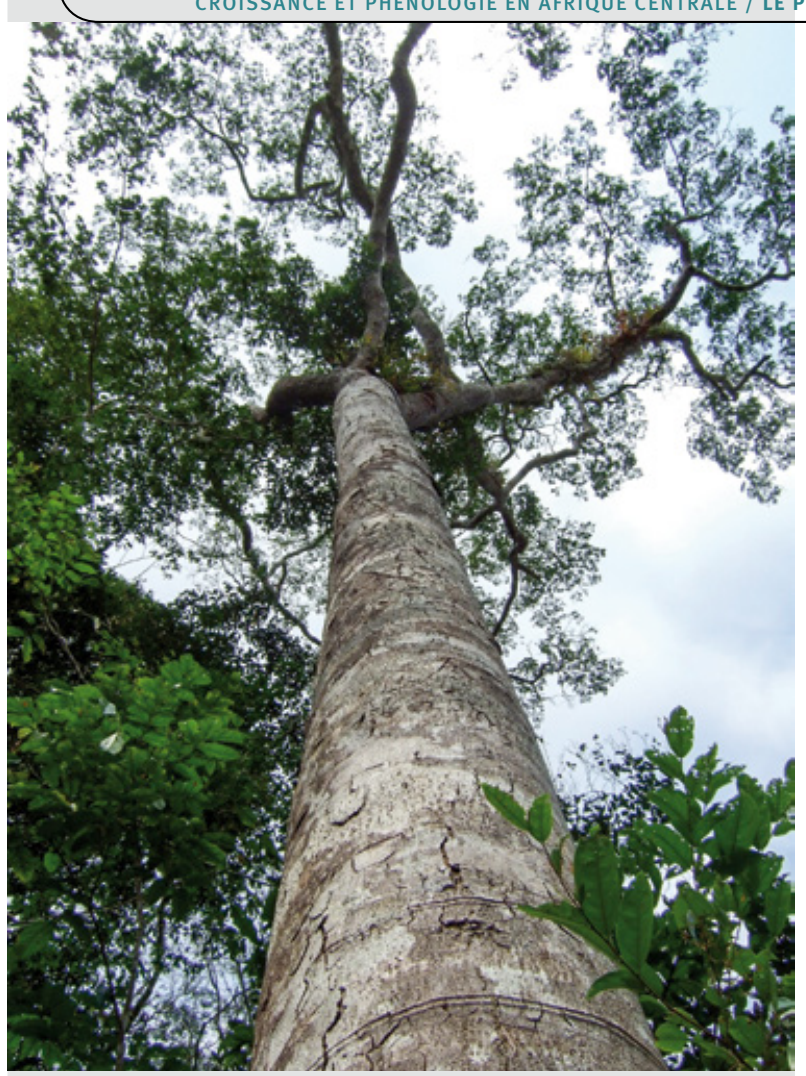

Erythrophleum suaveolens.

Photo N. Bourland.

distincte pour la première espèce et peu distincte pour les deux dernières (Detienne et al., 1998 ; Durrieu de Madron et al., 2000). En outre, dans la zone de démarcation des cernes (entre le bois final du cerne précédent et le bois initial du cerne suivant), d'autres particularités anatomiques telles que la variation de l'abondance du parenchyme axial ont été observées chez $E$. cylindricum, ou des vaisseaux chez $B$. toxisperma, ou encore de la taille des vaisseaux chez $E$. suaveolens (annexe 1). Ces observations corroborent les caractéristiques anatomiques retenues par Worbes (1995) dans la classification du caractère distinctif des cernes des arbres tropicaux. Ces résultats confirment également les observations de Detienne et al. (1998) selon lesquelles la présence et la visibilité des cernes dans le bois des arbres tropicaux dépendent plus du genre botanique que du climat, bien que ces caractères anatomiques ne soient pas nécessairement stables pour les taxa et restent variables selon les sites et les conditions de croissance (Fichtler et Worbes, 2012).

La présence et la lisibilité des cernes sont des préalables pour l'analyse dendrochronologique qui nécessite que chaque cerne soit associé à une année précise et mesurable avec précision. Pour les trois espèces étudiées, la présence de caractères anatomiques distincts et l'application de la méthode de marquage séquentiel préconisée par Mariaux (1967) ont permis de confirmer l'annualité de la formation des cernes (Detienne et al., 1998 ; Verheyden et al., 2004). La réaction cicatricielle au marquage réalisé chaque saison est variable selon l'espèce et la zone d'influence de la cicatrice est plus importante chez $E$. cylindricum et E. suaveolens que chez $B$. toxisperma (figures $2, C$ et D). Ces observations mettent ainsi en évidence les potentialités 


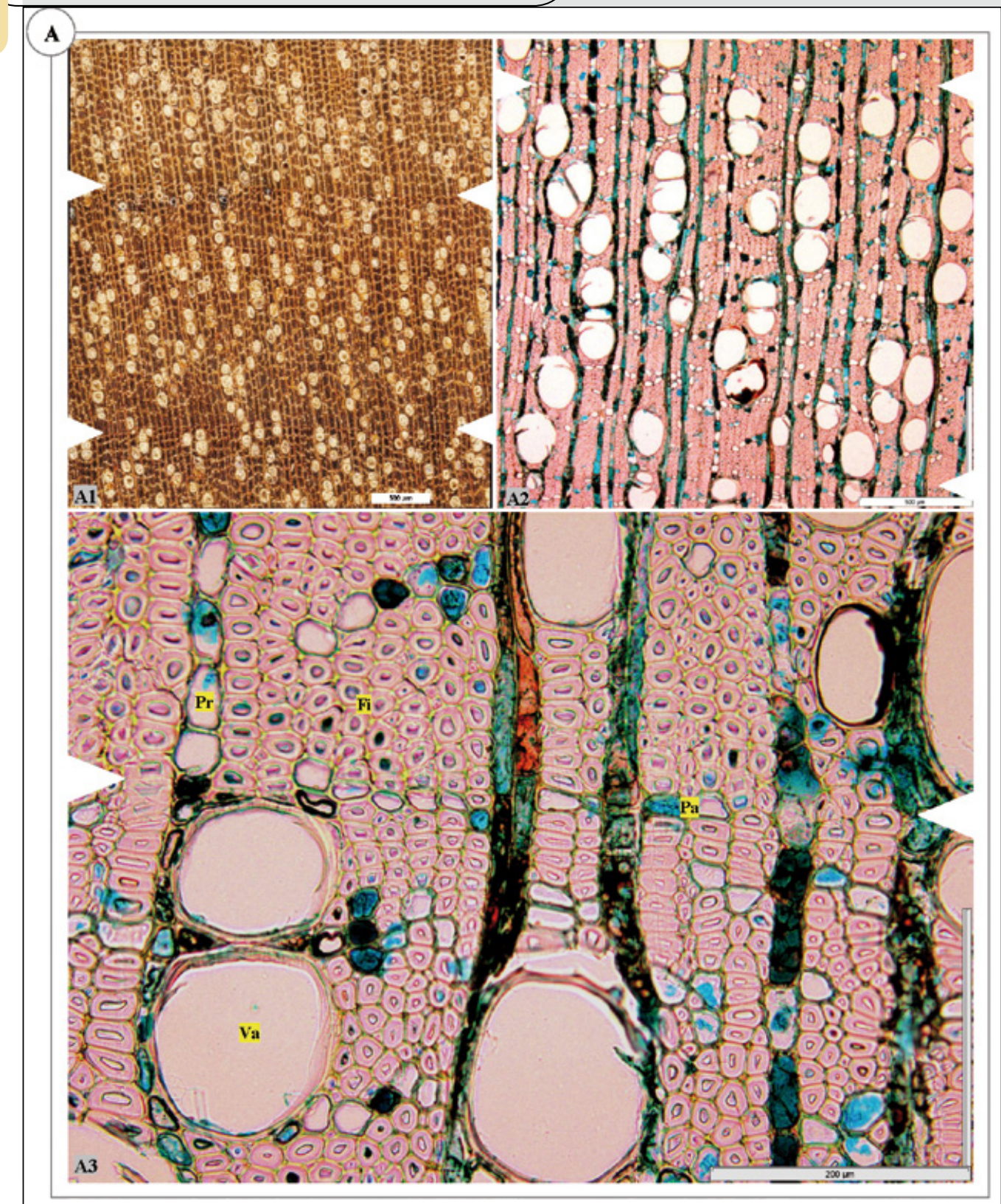

\section{Figure 4A.}

Anatomie macroscopique (A1) et microscopique ( $A 2$ et $A 3)$ de la section transversale du bois de Baillonella toxisperma. Les triangles en blanc indiquent la limite entre les cernes et la double flèche en noir indique la largeur de la couche de cellules aplaties de fibres précédant la ligne de parenchyme marginal délimitant le cerne. $\mathrm{Fi}$ : fibres ; Pa : parenchyme axial ; $\operatorname{Pr}:$ parenchyme radial; Va : vaisseaux. anatomiques de ces espèces permettant d'envisager l'analyse de leurs trajectoires de croissance, des données utiles à la détermination et à l'affinage des paramètres d'aménagement de leurs populations naturelles (Groenendijk et al., 2014 ; De Ridder et al., 2014). Néanmoins, les valeurs d'accroissement moyen périodique obtenues dans le cas de B. toxisperma sont bien plus faibles que celles observées par Fétéké et al. (2015) dans la même zone. Bien que les conditions environnementales, qui n'ont été quantifiées que de façon très sommaire (statut dominant et codominant des arbres), puissent avoir joué un rôle déterminant, le faible nombre d'individus étudiés ainsi que les stades ontogéniques ou phélonologiques entre les arbres pourraient également l'expliquer. Les valeurs obtenues dans le cadre de la présente étude ne peuvent en aucun cas être utilisées pour proposer des normes à utiliser pour le calcul des taux de reconstitution post-exploitation.

\section{Variabilité de la croissance}

La formation des cernes chez ces trois espèces est annuelle et se fait en grande partie pendant la période la plus humide de l'année s'étalant sur huit mois et représentant 78 à $91 \%$ de la largeur du cerne (tableau II). Ces résultats obtenus par la méthode de marquage cambial séquentiel corroborent d'autres études antérieures utilisant la méthode de mesure à l'aide d'un ruban dendrométrique pour d'autres espèces tropicales (Devineau, 1991 ; Lisi et al., 2008) ou l'interdatation et la datation par radiocarbone à l'aide d'isotopes pour les espèces tropicales dont la transition anatomique entre cernes est moins évidente (Trouet et al., 2001 ; Worbes et al., 2003).

On a pu néanmoins constater une variation entre les espèces, $B$. toxisperma se comportant mieux que les deux autres espèces pendant cette période de sécheresse, 


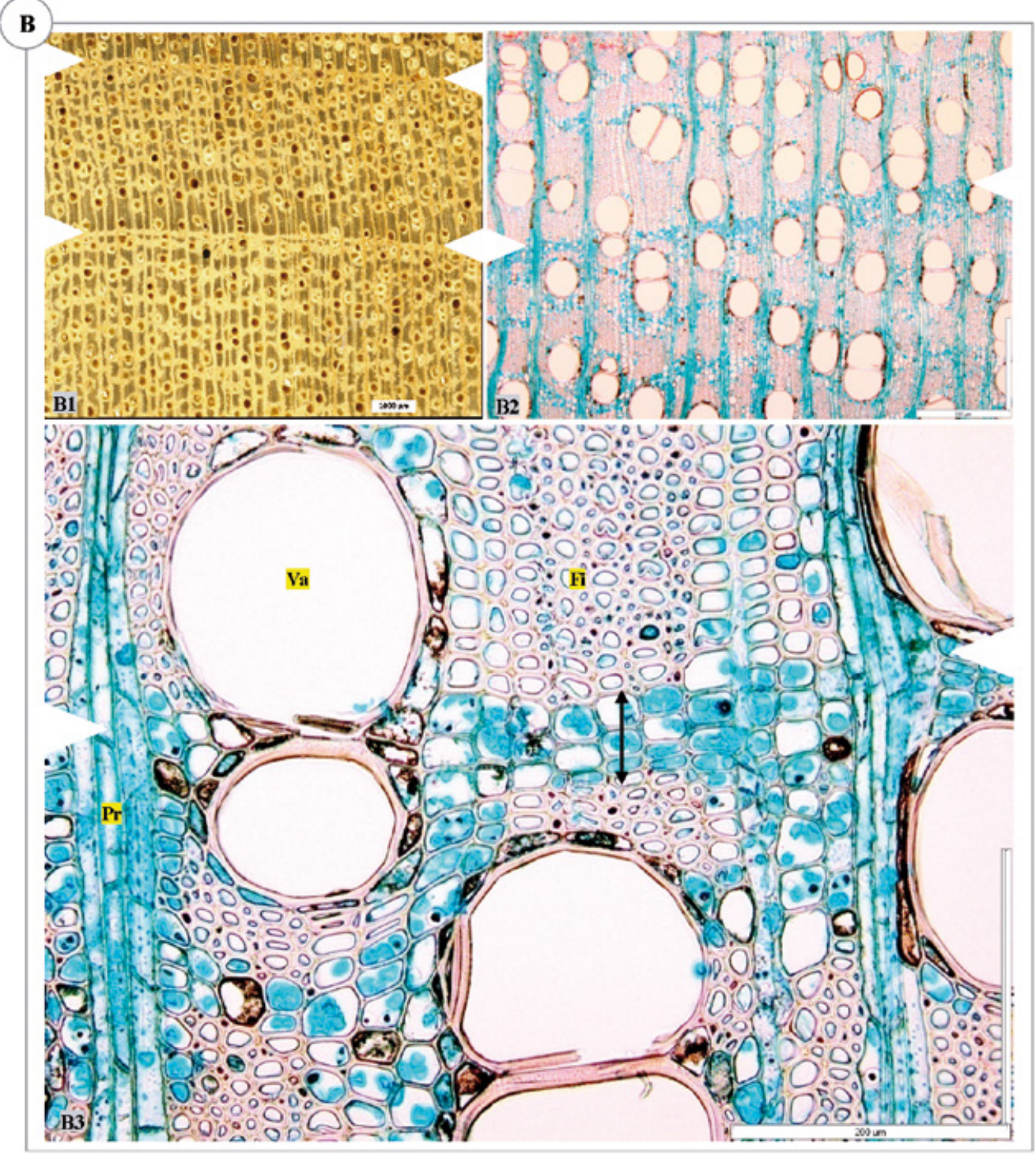

Figure 4B.

Anatomie macroscopique (B1) et microscopique (B2 et B3) de la section transversale du bois de Entandrophragma cylindricum. Les triangles en blanc indiquent la limite entre les cernes et la double flèche en noir indique la largeur de la couche de 3 à 4 cellules de parenchyme marginal qui délimite le cerne. Fi : fibres ; $\mathrm{Pa}$ : parenchyme axial ; Pr: parenchyme radial; Va : vaisseaux. témoignant ainsi de la diversité des potentialités d'adaptation chez les espèces tropicales (Ouédraogo et al., 2013). En revanche, la croissance relativement importante observée pendant les saisons les moins sèches de l'année (PSP, PSS et GSP), où la ressource en eau demeure abondante, pourrait résulter de l'effet significatif des précipitations sur les arbres au sortir d'une période sèche, comme observé chez des espèces tropicales amazoniennes (Devall et al., 1995) et d'Afrique centrale (Couralet et al., 2010 ; De Ridder et al., 2014). Néanmoins, le principal facteur climatique explicatif de la variation saisonnière de la croissance est la température (Tmoy) pour E. suaveolens, le rayonnement (Rmoy) jouant également un rôle dans la croissance de $B$. toxisperma. Ces résultats confirment dans leur ensemble les observations de Wagner et al. (2012) et Clark et al. (2010) relatives à l'influence directe de ces deux composantes climatiques sur la croissance des arbres tropicaux.

\section{Relations entre la croissance et les phénologies foliaire et reproductive}

Le climat influence également les phénologies foliaire et reproductive des trois espèces étudiées (corrélations significatives, annexe 3), à l'instar d'autres espèces tropicales (De Ridder et al., 2013, 2014). L'occurrence des phases foliaire et de reproduction chez B. toxisperma, E. cylindricum et $E$. suaveolens est déclenchée par l'intensité saisonnière des précipitations, de la température et de la lumière. La réponse des arbres à la variabilité saisonnière du climat diffère néanmoins d'une espèce à l'autre. Le rythme des événements phénologiques des arbres en milieu tropical est en effet connu pour répondre à des variations des conditions environnementales telles que l'apparition des premières pluies après une période de sécheresse (Williams et al., 2008) ou des changements de température (Yadav et Yadav, 2008) ou de l'irradiation (Bendix et al., 2006). Les variations de l'intensité de la lumière (Rmoy) et des précipitations 


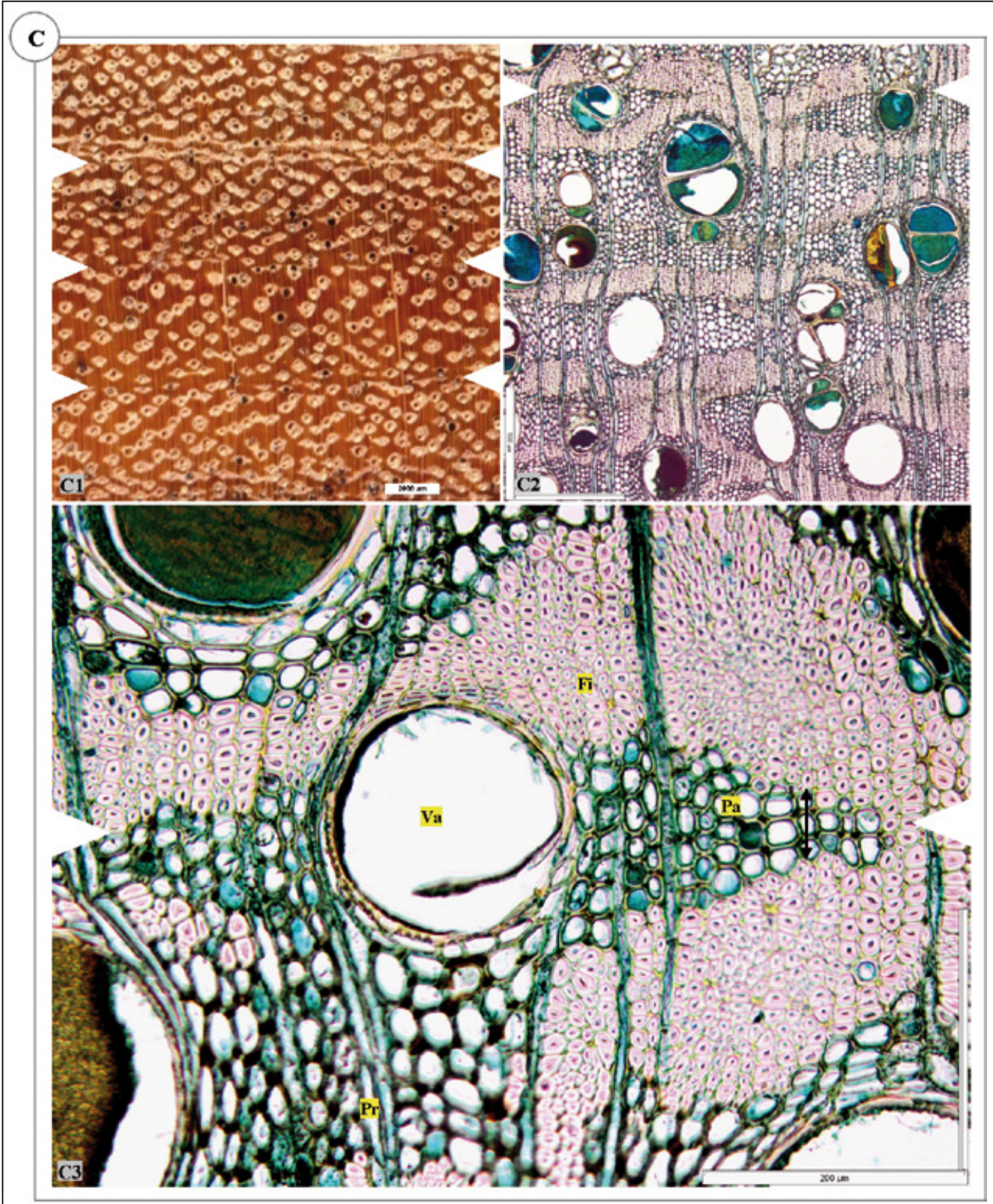

Figure 4C.

Anatomie macroscopique (C1) et microscopique (C2 et C3) de la section transversale du bois de Erythrophleum suaveolens. Les triangles en blanc indiquent la limite entre les cernes et la double flèche en noir indique la largeur de la couche discontinue de cellules de parenchyme marginal qui délimite le cerne. $\mathrm{Fi}$ : fibres ; $\mathrm{Pa}$ : parenchyme axial ; Pr: parenchyme radial : Va: vaisseaux.

(Pmoy) sont les principaux moteurs de la perte de feuilles et de l'apparition des fleurs chez $B$. toxisperma, de l'apparition des nouvelles feuilles et des fleurs chez $E$. cylindricum et de l'ensemble des événements phénologiques chez $E$. suaveolens (annexe 3). Ces observations abondent dans le sens de Myneni et al. (2007) qui ont observé que les radiations étaient le principal moteur de la défeuillaison des arbres en forêt amazonienne. Les variations de la température (Tmoy) affectent plutôt les phases de floraison et de fructification immature chez $E$. cylindricum et de défeuillaison/feuillaison chez E. suaveolens.

L'influence de la phénologie sur la croissance radiale demeure variable selon les espèces, ce qui confirme globalement les observations de Wagner et al. (2013) en Guyane. L'influence du recouvrement foliaire paraît néanmoins significative pour deux des espèces étudiées. La perte et le renouvellement des feuilles interviennent à une période où les précipitations sont les plus faibles et les températures ainsi que les radiations sont les plus élevées de l'année, ce qui correspond également à la période de faible croissance radiale. Pendant la petite saison des pluies, les précipitations et l'intensité des radiations relativement élevées, ainsi que la présence de feuillage abondant sur les arbres, constituent des conditions propices à la mobilisation de ressources suffisantes pour amorcer la fructification et surtout accroître la production de bois (Brando et al., 2010 ; De Weirdt et al., 2012). La diminution de la production de bois pendant les deux saisons suivantes (PSS et GSP, figure 3) pourrait s'expliquer d'une part par l'âge des arbres ainsi que la baisse de l'activité photosynthétique due au 


\section{Bibliographie}

vieillissement du feuillage, et d'autre part par la diminution de l'intensité de la lumière et des températures. L'âge des arbres pourrait également être un facteur explicatif de cette diminution. L'ensemble de ces observations expliquent que les arbres les mieux exposés à la lumière disposent davantage de ressources nécessaires pour assurer à la fois une meilleure reproduction (Menga et al., 2012) et un accroissement optimal de leur diamètre (Fétéké et al., 2015).

\section{Conclusion et perspectives}

L'étude porte sur trois espèces de forêt dense humide semi-caducifoliée du sud-est du Cameroun : Baillonella toxisperma Pierre (moabi, Sapotaceae), Entandrophragma cylindricum (Sprague) Sprague (sapelli, Meliaceae) et Erythrophleum suaveolens Guill. \& Perr. (tali, Fabaceae). Ces trois espèces présentent des cernes annuels visibles et distincts, et un rythme de croissance imprimé par les conditions climatiques locales. Ce potentiel anatomique, couplé à la sensibilité de ces espèces aux variations du climat, offre ainsi la possibilité de retracer leur trajectoire de croissance et constitue, de ce fait, une opportunité d'acquérir des données fiables et précieuses pour l'aménagement forestier. Dans le contexte actuel de la gestion forestière en Afrique centrale, basée sur le principe du rendement soutenu, ces données de croissance peuvent être directement utilisées pour estimer la production ligneuse ou pour évaluer la durabilité des prélèvements dans les forêts de production (Brienen et Zuidema, 2006). La corrélation entre la croissance radiale et les autres variables phénologiques et climatiques souligne l'adaptation de ces espèces aux conditions du milieu et met ainsi en évidence la nécessité de prendre en compte ces facteurs de variation dans les perspectives de modélisation de leur croissance. Enfin, une approche dendrochronologique beaucoup plus fine pourrait également permettre de mieux comprendre le développement des espèces ligneuses commerciales et des ressources que l'on peut en tirer de manière durable.

\section{Remerciements}

Les auteurs remercient la société Pallisco et le Musée royal de l'Afrique centrale (MRAC) pour leur soutien aussi bien logistique que technique dans la conduite de l'étude. Leurs remerciements vont également à l'endroit de Maaike De Ridder et Kevin Lievens pour leurs appuis et conseils avisés dans l'acquisition, l'analyse et l'interprétation des données anatomiques. Les auteurs remercient également le projet DynAfFor financé par le Fonds français pour l'environnement mondial (FFEM) et l'Agence française de développement (AFD).
Bendix J., Homeier J., Cueva E. O., Emck P., Breckle S.-W., Richter M. et al., 2006. Seasonality of weather and tree phenology in a tropical evergreen mountain rain forest. International Journal of Biometeorology, 50: 370-384.

Bibani Mbarga R., Jonkers W. B. J., Essama Etoundi J., 1999. Phénologie de 86 essences productrices de bois d'œuvre de la forêt dense humide sempervirente du Sud-Cameroun - Résultats préliminaires. In : Nasi R., Amsallem I., Drouineau S. (éds). La gestion des forêts denses africaines aujourd'hui : Actes du séminaire Forafri de Libreville, Gabon. Montpellier, France, Cirad-Forêt, 16 p. CD-Rom.

Bourland N., Kouadio Y. L., Lejeune P., Bonnaventure S., Philippart J., Daïnou K., Fétéké F. R., Doucet J.-L., 2012. Ecology of Pericopsis elata (Fabaceae), an endangered timber species in southeastern Cameroon. Biotropica, 44: 840-847.

Brando P. M., Goetz S. J., Baccini A., Nepstad D. C., Beck P. S. A., Christman M. C., 2010. Seasonal and inter-annual variability of climate and vegetation indices across the Amazon. Proceedings of the National Academy of Sciences, 107: 14685-14690.

Brienen R. J. W., Zuidema P. A., 2006. The use of tree rings in tropical forest management: Projecting timber yields of four Bolivian tree species. Forest Ecology and Management, 226: 256-267.

Cardoso F. C. G., Marques R., Botosso P. C., Marques M. C. M., 2012. Stem growth and phenology of two tropical trees in contrasting soil conditions. Plant Soil, 354: 269-281.

Clark D. B., Clark D. A., Oberbauer S. F., 2010. Annual wood production in a tropical rain forest in N.E. Costa Rica linked to climatic variation but not to increasing $\mathrm{CO}_{2}$. Global Change Biology. 16: 747-759.

Couralet C., Sterck F. J., Sass-Klaassen U., Van Acker J., Beeckman H., 2010. Species-Specific Growth Responses to Climate Variations in Understory Trees of a Central African Rain Forest: Climate-Growth Relationships of Understory Trees. Biotropica, 42: 503-511.

Davies S. J., Ashton P. S., 1999. Phenology and fecundity in 11 sympatric pioneer species of Macaranga (Euphorbiaceae) in Borneo. American Journal of Botany, 86: 1786-1795.

De Ridder M., Toirambe B., Van den Bulcke J., Bourland N., Van Acker J., Beeckman H., 2014. Dendrochronological Potential in a Semi-Deciduous Rainforest: The Case of Pericopsis elata in Central Africa. Forests, 5: 3087-3106.

De Ridder M., Van den Bulcke J., Van Acker J., Beeckman H., 2013. Tree-ring analysis of an African long-lived pioneer species as a tool for sustainable forest management. Forest Ecology and Management, 304: 417-426. 
Detienne P., Mariaux A., 1977. Nature et périodicité des cernes dans les bois rouges de méliacées africaines. Bois Tropicaux, 175 : 52-61.

Detienne P., Oyono F., Durrieu de Madron L., Demarquez B., Nasi R., 1998. L'analyse des cernes : applications aux études de croissance de quelques essences en peuplements naturels de forêt dense africaine. Montpellier, France, Cirad, série Forafri, $n^{\circ} 15$. CD-Rom.

Devall M. S., Parresol B. R., Wright S. J., 1995. Dendroecological analysis of Cordia alliodora, Pseudobombax septenatum and Annona spraguei in central Panama. IAWA Journal, 16: 411-424.

Devineau J.-L., 1991. Variabilité de la croissance en circonférence des arbres dans les forêts semi-décidues de Lamto (Côte d'Ivoire). La Terre et la Vie - Revue d’Écologie, 46 : 95-123.

De Wasseige C., De Marcke P., Bayol N., Hiol Hiol F., Mayaux P., Desclée B., Nasi R., Billand A., Defourny P., Eba'a Atyi R., 2012. Les forêts du bassin du Congo. État des Forêts 2010. Luxembourg, Office des publications de l'Union européenne, $276 \mathrm{p}$.

De Weirdt M., Verbeeck H., Maignan F., Peylin P., Poulter B., Bonal D. et al., 2012. Seasonal leaf dynamics for tropical evergreen forests in a process-based global ecosystem model. Geoscientific Model Development, 5: 1091-1108.

Dié A., Kitin P., Kouame F. N., Van den Bulcke J., Van Acker J., Beeckman H., 2012. Fluctuations of cambial activity in relation to precipitation result in annual rings and intra-annual growth zones of xylem and phloem in teak (Tectona grandis) in Ivory Coast. Annals of Botany, 110: 861-873.

Durrieu de Madron L., Daumerie A., 2004. Diamètre de fructification de quelques essences en forêt naturelle centrafricaine. Bois et Forêts des Tropiques, $281: 87-95$. http://bft. cirad.fr/cd/BFT 281 87-95.pdf

Durrieu de Madron L., Forni E., 1997. Aménagement forestier dans l'est du Cameroun : Structure du peuplement et périodicité d'exploitation. Bois et Forêts des Tropiques, 254 : 39-50. http://bft.cirad.fr/cd/BFT 254 39-50.pdf

Durrieu de Madron L., Nasi R., Detienne P., 2000. Accroissements diamétriques de quelques essences en forêt dense africaine. Bois et Forêts des Tropiques, 263 : 63-74. http:// bft.cirad.fr/cd/BFT 263 63-74.pdf

Fayolle A., Swaine M. D., Bastin J.-F., Bourland N., Comiskey J. A., Dauby G. et al., 2014. Patterns of tree species composition across tropical African forests. Journal of Biogeography, 41 (12): 2320-2331.

Fétéké F., Perin J., Fayolle A., Daïnou K., Bourland N., Kouadio Y. L. et al., 2015. Modéliser la croissance de quatre essences pour améliorer la gestion forestière au Cameroun. Bois et Forêts des Tropiques, $325: 5-20$. http://bft.cirad.fr/ cd/BFT 325 5-20.pdf
Fichtler E., Worbes M., 2012. Wood anatomical variables in tropical trees and their relation to site conditions and individual tree morphology. IAWA Journal, 33 (2): 119-140.

Groenendijk P., Sass-Klaassen U., Bongers F., Zuidema P. A., 2014. Potential of tree-ring analysis in a wet tropical forest: A case study on 22 commercial tree species in Central Africa. Forest Ecology and Management, 323: 65-78.

Jacoby G. C., 1989. Overview of Tree-Ring Analysis in Tropical Regions. IAWA Journal, 10: 99-108.

Jammalamadaka S. R., SenGupta A., 2001. Topics in Circular Statistics. World Scientific, Series on Multivariate Analysis, vol. 5.

Jones A., Breuning-Madsen H., Brossard M., Dampha A., Deckers J., Dewitte O. et al., 2013. Soil Atlas of Africa. Luxembourg, European Commission, Publications Office of the European Union, 176 p.

Kariuki M., Rolfe M., Smith R. G. B., Vanclay J. K., Kooyman R. M., 2006. Diameter growth performance varies with species functional-group and habitat characteristics in subtropical rainforests. Forest Ecology and Management, 225: 1-14.

Latte N., Bauwens S., Bonnet S., Lejeune P., Fayolle A., 2014. Dendrochronological analysis of large tropical trees: a new approach combining photogrammetry, image processing and GIS tools. 9th International Conference on Dendrochronology, January 13-17, 2014, Melbourne, Australia.

Letouzey R., 1986. Carte phytogéographique du Cameroun au 1: 500.000 (1985) : Domaine de la forêt dense humide semi-caducifoliée. SC. III. - Notices de la carte. Toulouse, France, Institut de la carte internationale de la végétation.

Lisi C. S., Fo M. T., Botosso P. C., Roig F. A., Maria V. R., Ferreira-Fedele L. et al., 2008. Tree-ring formation, radial increment periodicity, and phenology of tree species from a seasonal semi-deciduous forest in Southeast Brazil. IAWA Journal, 29: 189-207.

Mariaux A., 1967. Les cernes dans les bois tropicaux africains : nature et périodicité. Bois et Forêts des Tropiques, 114 : 23-37. http://bft.cirad.fr/cd/BFT 114 23-37.pdf

Martin D., Sieffermann G., Vallerie M., 1966. Les sols rouges du Nord Cameroun. Cahiers Orstom, série Pédologie, 4 (3), $196 \mathrm{p}$.

Menga P., Bayol N., Nasi R., Fayolle A., 2012. Phénologie et diamètre de fructification du wengé, Millettia laurentii De Wild. : implications pour la gestion. Bois et Forêts des Tropiques, 312 : 31-41. http://bft.cirad.fr/cd/BFT 312 31-41. pdf

Meunier Q., Moumbogou C., Doucet J.-L., 2015. Les arbres utiles du Gabon. Gembloux, Belgique, Presses agronomiques de Gembloux, 340 p. 
Morel H., Mangenet T., Beauchêne J., Ruelle J., Nicolini E., Heuret P., 2015. Seasonal variations in phenological traits: leaf shedding and cambial activity in Parkia nitida Miq. and Parkia velutina Benoist (Fabaceae) in tropical rainforest. Trees, 29: 973-984.

Morellato L. P. C., Camargo M. G. G., Neves F. F. D., Luize B. G., Mantovani A., Hudson I. L., 2010. The Influence of Sampling Method, Sample Size, and Frequency of Observations on Plant Phenological Patterns and Interpretation in Tropical Forest Trees. In: Hudson I. L., Keatley M. R. (eds). Phenological Research. Methods for Environmental and Climate Change Analysis. Dordrecht, Netherlands, Springer, 99-121.

Myneni R. B., Yang W., Nemani R. R., Huete A. R., Dickinson R. E., Knyazikhin Y. et al., 2007. Large seasonal swings in leaf area of Amazon rainforests. Proceedings of the National Academy of Sciences, 104: 4820-4823.

Narboni P., 2000. Bases de données et forêts naturelles. Les dispositifs de recherche et les inventaires forestiers. Bois et Forêts des Tropiques, $265:$ 21-30. http://bft.cirad.fr/cd/ BFT 265 21-30.pdf

Newstrom L. E., Frankie G. W., Baker H. G., Colwell R. K., 1994. Diversity of long-term flowering patterns. In: Mc Dade L. A., Bawa K. S., Hespenhaide H. A., Hartshorn G. S. (eds). La Selva: Ecology and Natural History of a Neotropical Rain Forest. University of Chicago, USA, 143-159.

Nzogang A., 2009. Tropical forest dynamics after logging: natural regeneration and growth of commercial tree species in southeast Cameroon. Faculty of Forest and Environmental Sciences, Albert-Ludwigs-Universität, Freibourg, Germany.

Ouédraogo D.-Y., Mortier F., Gourlet-Fleury S., Freycon V., Picard N., 2013. Slow-growing species cope best with drought: evidence from long-term measurements in a tropical semi-deciduous moist forest of Central Africa. Journal of Ecology, 101: 1459-1470.

Picard N., Gourlet-Fleury S., 2008. Manuel de référence pour l'installation de dispositifs permanents en forêt de production dans le Bassin du Congo. Yaoundé, Cameroun, COMIFAC

R Development Core Team, 2013. R: A language and environment for statistical computing. Vienna, Austria, R Foundation for Statistical Computing.

Reed S. C., Wood T. E., Cavaleri M. A., 2012. Tropical forests in a warming world. New Phytologist, 193: 27-29.

Reich P. B., 1995. Phenology of tropical forests: patterns, causes, and consequences. Canadian Journal of Botany, 73 : 164-174.

Richardson A. D., Keenan T. F., Migliavacca M., Ryu Y., Sonnentag O., Toomey M., 2013. Climate change, phenology, and phenological control of vegetation feedbacks to the climate system. Agricultural and Forest Meteorology, 169: 156-173.
Schöngart J., Piedade M. T. F., Ludwigshausen S., Horna V., Worbes M., 2002. Phenology and stem-growth periodicity of tree species in Amazonian floodplain forests. Journal of Tropical Ecology, 18 (4): 581-597.

Stahle D. W., Mushove P. T., Cleaveland M. K., Roig F., Haynes G. A., 1999. Management implications of annual growth rings in Pterocarpus angolensis from Zimbabwe. Forest Ecology and Management, 124: 217-229.

Suchel J. B., 1988. Les climats du Cameroun. Thèse doctorat d’État, Université de Saint-Étienne, France, 4 vol., 1188 p.

Trouet V., Haneca K., Coppin P., Beeckman H., 2001. Tree ring analysis of Brachystegia spiciformis and Isoberlina tomentosa: an evaluation of the ENSO-signal in the Miombo woodland of eastern Africa. IAWA Journal, 22: 385-399.

Verheyden A., Kairo J. G., Beeckman H., Koedam N., 2004. Growth rings, Growth ring formation and age determination in the mangrove Rhizophora mucronata. Annals of Botany, 94: 59-66.

Wagner F., Rossi V., Aubry-Kientz M., Bonal D., Dalitz H., Gliniars R. et al., 2014. Pan-Tropical Analysis of Climate Effects on Seasonal Tree Growth. PLoS ONE, 9: e92337.

Wagner F., Rossi V., Stahl C., Bonal D., Hérault B., 2013. Asynchronism in leaf and wood production in tropical forests: a study combining satellite and ground-based measurements. Biogeosciences, 10: 7307-7321.

Wagner F., Rossi V., Stahle C., Bonal D., Hérault B., 2012. Water Availability Is the Main Climate Driver of Neotropical Tree Growth. PLoS ONE, 7: e34074.

Wheeler E. A., 2011. InsideWood - a web resource for hardwood anatomy. IAWA Journal, 32: 199-211.

White F., 1983. The vegetation of Africa. Brittonia, 36: 273-273.

Williams L. J., Bunyavejchewin S., Baker P. J., 2008. Deciduousness in a seasonal tropical forest in western Thailand: inter-annual and intra-specific variation in timing, duration and environmental cues. Fcologia, 155: 571-582.

Worbes M., 1995. How to Measure Growth Dynamics in Tropical Trees a Review. IAWA Journal, 16: 337-351.

Worbes M., Fichtler E., 2010. Wood anatomy and tree-ring structure and their importance for tropical dendrochronology. In: Junk W. J., Piedade M. T. F., Wittmann F., Schöngart J., Parolin P. (eds). Amazonian Floodplain Forests. Dordrecht, Netherlands, Springer, 329-346.

Worbes M., Staschel R., Roloff A., Junk W. J., 2003. Tree ring analysis reveals age structure, dynamics and wood production of a natural forest stand in Cameroon. Forest Ecology and Management, 173: 105-123.

Yadav R. K., Yadav A. S., 2008. Phenology of selected woody species in a tropical dry deciduous forest in Rajasthan, India. Tropical Ecology, 49: 25-34. 


\section{Annexe 1. \\ Description anatomique des espèces (Wheeler, 2011).}

\section{Moabi, Baillonella toxisperma Pierre (figure 4A)}

$2 \mathrm{v} \quad$ Limite de cerne visible ou indistincte : (c) parenchyme marginal en fil irrégulier associé à une modification de la structure des fibres (aplatissement) dans le bois final précédant une large couche de fibres pauvre en vaisseaux et en parenchyme dans le bois initial du cerne suivant

$5 \quad$ Bois à pores diffus

$7 \mathrm{v} \quad$ Disposition des vaisseaux en file radiale ou en diagonale

10v Regroupement des vaisseaux : uniques ou accolés par multiples de 3 à 4

12 Vaisseaux à contours arrondis

42 Taille des vaisseaux : gros vaisseaux de 103-199 $\mu \mathrm{m}$

47 Densité des vaisseaux : 7-15 vaisseaux par $\mathrm{mm}^{2}$ (rares)

66 Fibres non cloisonnées

$70 \quad$ Fibres à paroi épaisse

77v Parenchyme axial apotrachéal (non associé à des vaisseaux) diffus en chaînettes

86 Parenchyme axial en bande d'une cellule

87 Parenchyme axial disposé en réseau

\section{Sapelli, Entandrophragma cylindricum (Sprague) Sprague (figure 4B)}

$1 \mathrm{v} \quad$ Limite de cerne visible et distincte : (c) parenchyme marginal terminal en bande continue de 3 à 7 cellules de large séparant une zone distincte de fibres du bois initial du cerne suivant

$5 \quad$ Bois à pore diffus

$7 v \quad$ Disposition des vaisseaux en file radiale ou oblique

10 Regroupement des vaisseaux : isolés ou accolés par paire

12 Contour arrondi des vaisseaux

42 Taille des vaisseaux : gros vaisseaux de 108-195 $\mu \mathrm{m}$

$47 \quad 10-15$ vaisseaux par $\mathrm{mm}^{2}$ (rare)

69 Fibres à paroi d'épaisseur moyenne

78 Parenchyme axial paratrachéal juxtavasculaire

79v Parenchyme axial paratrachéal en manchon

80 Parenchyme axial paratrachéal aliforme

81v Parenchyme axial paratrachéal aliforme en losange

82v Parenchyme axial paratrachéal aliforme ailé

83 Parenchyme axial paratrachéal confluent

85 Parenchyme axial en bande de plus de 3 à 7 cellules

89 Parenchyme axial en bande marginale

131 Présence de canaux traumatiques

\section{Tali, Erythrophleum suaveolens Guill. \& Perr. (figure 4C)}

2 Limite de cerne indistincte : (c) parenchyme marginal terminal formant en bande fine discontinue avec des extensions latérales autour des vaisseaux de taille réduite.

$5 \quad$ Bois à pores diffus (taille des vaisseaux presque identique)

6 Vaisseaux disséminés

10v Disposition partiellement isolée des vaisseaux ou accolée radialement par 2

12 Vaisseaux à contours arrondis

42 Taille des vaisseaux : moyen à gros $(55-195 \mu \mathrm{m})$

$45 \quad$ Présence de vaisseaux de deux tailles distinctes

$46 \quad 3$ à 5 vaisseaux par $\mathrm{mm}^{2}$ (très rares)

$47 \mathrm{v} \quad 3$ à 7 vaisseaux par $\mathrm{mm}^{2}$ (rares)

$70 \quad$ Fibres à paroi épaisse

80 Parenchyme axial aliforme losangique

83 Parenchyme axial paratrachéal anastomosé

\section{Annexe 2.}

Statistiques descriptives du climat de la zone d'étude sur la période 2010-2013.

Statistiques

Moyenne

Coefficient de variation

\begin{tabular}{|c|}
\hline Précipitations \\
\hline 1490,85 \\
\hline 4,91
\end{tabular}

Interannuel

Température

24,34

0,85

4,26

Radiation

Précipitations

Intersaison

Température

Radiation

117,13

53,18

24,35

17,54 


\section{Annexe 3.}

Corrélations entre variables de croissance, phénologiques et climatiques saisonnières. Les chiffres en gras indiquent une forte colinéarité (corrélation significative) entre les variables d'une même catégorie. RFol: taux de recouvrement par les feuilles; RFeu : taux de recouvrement par les nouvelles feuilles; RFlo : taux de recouvrement par les fleurs ; RFri : taux de recouvrement par les fruits immatures ; RFrm : taux de recouvrement par les fruits matures ; Pmoy : moyennes des précipitations ; Rmoy : moyennes des radiations ; Tmoy : moyenne de température.

\begin{tabular}{|c|c|c|c|c|c|c|c|c|c|}
\hline $\begin{array}{l}\text { Variables } \\
\text { Moabi } \\
\text { Baillonello } \\
\mathrm{ACC}_{\mathrm{s}}\end{array}$ & $\begin{array}{c}\qquad A c c_{s} \\
\text { perma Pier }\end{array}$ & RFol & RFeu & RFlo & RFri & RFrm & Pmoy & Rmoy & Tmoy \\
\hline RFol & $-0,81^{(* *)}$ & & & & & & & & \\
\hline RFeu & $-0,08^{(\mathrm{ns})}$ & $0,06^{\text {(ns) }}$ & & & & & & & \\
\hline RFlo & $0,28^{(\mathrm{ns})}$ & $-0,66^{(\mathrm{ns})}$ & $-0,52^{(\mathrm{ns})}$ & & & & & & \\
\hline RFri & $-0,14^{(\mathrm{ns})}$ & $0,18^{\text {(ns) }}$ & $0,99^{(\star \star)}$ & $-0,63^{\text {(ns) }}$ & & & & & \\
\hline RFrm & $-0,63^{(n s)}$ & $0,49^{\text {(ns) }}$ & $0,77^{(\star \star)}$ & $-0,47^{(n s)}$ & $0,80^{(\star *)}$ & & & & \\
\hline Pmoy & $-0,26^{(n s)}$ & $0,73^{\text {(ns) }}$ & $0,01^{\text {(ns) }}$ & $-0,85^{(\star \star \star)}$ & $0,14^{(\mathrm{ns})}$ & $0,05^{\text {(ns) }}$ & & & \\
\hline Rmoy & $0,79^{(\star \star)}$ & $-0,88^{(\star *)}$ & $-0,49^{(n s)}$ & $0,74^{(*)}$ & $-0,58^{(\mathrm{ns})}$ & $-0,84^{(\star *)}$ & $-0,54^{(\mathrm{ns})}$ & & \\
\hline Tmoy & $0,74^{(*)}$ & $-0,53^{(\mathrm{ns})}$ & $0,47^{(n s)}$ & $-0,26^{(n s)}$ & $0,43^{(n s)}$ & $-0,19^{(\mathrm{ns})}$ & $0,06^{(n s)}$ & $0,35^{(\mathrm{ns})}$ & \\
\hline
\end{tabular}

\section{Sapelli}

Entandrophragma cylindricum (Sprague) Sprague

$\mathrm{ACC}_{\mathrm{s}}$

\begin{tabular}{|c|c|c|c|c|c|c|c|c|}
\hline RFol & $0,61^{(* \star)}$ & & & & & & & \\
\hline RFeu & $-0,08^{(\mathrm{ns})}$ & $-0,45^{(*)}$ & & & & & & \\
\hline RFlo & $-0,35^{\text {(ns) }}$ & $-0,76^{(\star \star \star)}$ & $0,86^{(\star \star \star)}$ & & & & & \\
\hline RFri & $0,79^{(\star \star *)}$ & $0,72^{(\star \star)}$ & $-0,02^{(\mathrm{ns})}$ & $-0,42^{(*)}$ & & & & \\
\hline RFrm & $-0,03^{(\mathrm{ns})}$ & $0,45^{(*)}$ & $-0,63^{(\star \star \star)}$ & $-0,41^{(*)}$ & $-0,20^{(n s)}$ & & & \\
\hline Pmoy & $0,20^{\text {(ns) }}$ & $0,31^{\text {(ns) }}$ & $-0,79^{(* \star)}$ & $\left.-0,81^{(\star \star}\right)$ & $0,27^{\text {(ns) }}$ & $0,02^{(n s)}$ & & \\
\hline Rmoy & $0,11^{\text {(ns) }}$ & $-0,35^{\text {(ns) }}$ & $0,94^{(\star \star *}$ & $0,70^{(\star \star \star)}$ & $0,24^{\text {(ns) }}$ & $-0,82^{(\star \star \star)}$ & $-0,54^{(\star *)}$ & \\
\hline Tmoy & $0,78^{(\star \star \star)}$ & $0,67^{(\star \star)}$ & $0,13^{\text {(ns) }}$ & $-0,25^{(n s)}$ & $0,97^{(\star \star \star)}$ & $-0,18^{(n s)}$ & $0,06^{(\mathrm{ns})}$ & $0,35^{\text {(ns) }}$ \\
\hline
\end{tabular}

Tali

Erythrophleum suaveolens Guill. \& Perr.

$\mathrm{ACC}_{\mathrm{s}}$

RFol $\quad 0,36^{\text {(ns) }}$

RFeu $\quad 0,34^{\text {(ns) }}-0,26^{\text {(ns) }}$

RFlo $\quad-0,06^{(\mathrm{ns})}-0,56^{(*)} \quad 0,47^{(*)}$

RFri $\quad 0,28^{(\mathrm{ns})} \quad \mathbf{0 , 9 6 ^ { ( * \star ) }}-0,36^{(\mathrm{ns})}-0,65^{(* \star *)}$

RFrm $\quad-0,07^{(\mathrm{ns})} \quad 0,53^{(* *)}-0,61^{(\star *)}-0,38^{(\mathrm{ns})}$

Pmoy

$0,14^{(n s)} \quad 0,75^{(* \star)}$

Rmoy

Tmoy

$\begin{array}{cc}0,08^{(\mathrm{ns})} & -0,66^{(\star \star *)} \\ 0,55^{(\star *)} & 0,42^{(*)}\end{array}$

$-0,53^{(* \star)}$

$-0,59^{(\star \star)} \quad 0,63^{(\star \star *} \quad 0,19^{(\mathrm{ns})}$

$0,82^{(* \star)} \quad 0,54^{(* *)}-0,79^{(* \star)}-0,91^{(* \star)}-0,54^{(* *)}$

$0,74^{(\star \star)} \quad-0,12^{\text {(ns) }} \quad 0,30^{\text {(ns) }}-0,28^{(n s)} 0,06^{(n s)} 0,35^{(n s)}$

Significativité des corrélations : (ns) = non significatif $;\left(^{\star}\right) \leq 0,05 ;\left(^{(\star}\right) \leq 0,01 ;\left(^{(\star \star}\right) \leq 0,001$. 